\title{
NOMALIZERS OF NONNORMAL SUBGROUPS OF FINITE $p$-GROUPS
}

\author{
Qinhai Zhang and Juan GaO
}

\begin{abstract}
Assume $G$ is a finite $p$-group and $i$ is a fixed positive integer. In this paper, finite $p$-groups $G$ with $\left|N_{G}(H): H\right|=p^{i}$ for all nonnormal subgroups $H$ are classified up to isomorphism. As a corollary, this answers Problem 116(i) proposed by Y. Berkovich in his book "Groups of Prime Power Order Vol. I" in 2008.
\end{abstract}

\section{Introduction}

Assume $G$ is a group and $H$ is a subgroup of $G$. A simple fact is that $H \triangleleft G$ if and only if $N_{G}(H)=G$. $H$ is called self-normalizing if $N_{G}(H)=H ; H$ is called an abnormal subgroup if $g \in\left\langle H, H^{g}\right\rangle$ for all $g \in G$. R. W. Carter [3] proved an abnormal subgroup must be a self-normalizing. Obviously, the concept of abnormal subgroups (self-normalizing) is an extreme case of normal subgroups. A. Fattahi [4] determined finite groups with normal and abnormal subgroups (self-normalizing). Since then, Zhang [11, 12, 13, 14] replaced the condition "normal" in [4] by quasinormal, $s$-quasinormal, seminormal and $s$-seminormal, respectively, and determined finite groups with quasinormal ( $s$ quasinormal, seminormal and $s$-seminormal, respectively) and abnormal subgroups (self-normalizing).

It is natural to ask that if the condition "self-normalizing" in [4] is replaced by " $N_{G}(H): H \mid=p_{1} p_{2} \cdots p_{s}$ ", where $p_{i}$ is a prime and $s$ is a positive integer, then what can be said about finite groups $G$ with $\left|N_{G}(H): H\right|=p_{1} p_{2} \cdots p_{s}$ for nonnormal subgroups $H$ ? It turned out that such groups must be groups of prime power order, i.e., finite $p$-groups. In this paper, we classified finite $p$-groups $G$ with $\left|N_{G}(H): H\right|=p^{i}$ for nonnormal subgroups $H$, where $i$ is a fixed positive integer. As a corollary, this answers Problem 116(i) proposed by Y. Berkovich in his book "Groups of Prime Power Order Vol. I" in 2008.

Received October 21, 2010 ; Revised November 25, 2010.

2010 Mathematics Subject Classification. Primary 20D15, 20 E99.

Key words and phrases. finite p-groups, nonnormal subgroups, self-normalizer, central extensions.

This work was supported by NSFC (No. 11071150), by NSF of Shanxi Province (No. 2008012001) and The Returned Abroad-Student Fund of Shanxi Province (No. [2011]8-59). 
Problem 116(i). Classify the $p$-groups such that $\left|N_{G}(H): H\right|=p$ for all nonnormal subgroups $H<G$.

For convenience, we introduce the following symbols.

$\mathcal{S}_{1}=\left\{G \mid G\right.$ with $\left|N_{G}(H): H\right|=p$ for nonnormal subgroups $H$ of $\left.G\right\}$;

$\mathcal{S}_{2}=\left\{G \mid G\right.$ with $\left|N_{G}(H): H\right|=p^{2}$ for nonnormal subgroups $H$ of $\left.G\right\}$;

$\mathcal{S}_{3}=\left\{G \mid G\right.$ with $\left|N_{G}(H): H\right|=p^{i}$ for nonnormal subgroups $H$ of $G$, $i \geq 3\}$

$G_{n}$ denotes the $n$th term of the lower central series of a groups $G$. $M \lessdot G$ denotes $M$ is a maximal subgroup of a group $G$. In this paper $G$ denotes a finite $p$-group.

Let $G$ be a finite $p$-group. For a positive integer $i$, we define $\Omega_{i}(G)=\langle a \in$ $G\left|a^{p^{i}}=1\right\rangle$, and $\mho_{i}(G)=\left\langle a^{p^{i}} \mid a \in G\right\rangle$.

\section{Preliminaries}

Definition. Assume $G$ is a finite nonabelian group. $G$ is called minimal nonabelian if every proper subgroup of $G$ is abelian; $G$ is said to be a meta-Hamilton group if every proper subgroup of $G$ is abelian or normal. A subgroup $H$ of a group $G$ is called fully-normal if $K \unlhd G$ provided $K \leq H$.

Definition. Assume $A$ and $B$ are subgroups of a group $G$. If $G=A B$ and $[A, B]=1$, then $G$ is called a central product of $A$ and $B$, denoted by $A * B$.

Definition. Assume that $\mathcal{P}$ is a group theoretic property. $\mathcal{P}$ is called inheritable by subgroups if a group $G$ is a $\mathcal{P}$-group, then every subgroup $H$ of $G$ is also a $\mathcal{P}$-group; $\mathcal{P}$ is called inheritable by quotient groups if a group $G$ is a $\mathcal{P}$-group, then every quotient group $G / N$ is also a $\mathcal{P}$-group.

Definition. Assume $G$ is a group of order $p^{n}, n \geq 2$. $G$ is called a group of maximal class if $c(G)=n-1 ; G$ is called metaabelian if $G^{\prime \prime}=1 ; G$ is called metacyclic if $G$ has a cyclic normal subgroup $N$ such that $G / N$ is cyclic; $G$ is called $p^{s}$-abelian if $(a b)^{p^{s}}=a^{p^{s}} b^{p^{s}}$ for any $a, b \in G$, where $s$ is a positive integer.

Lemma 2.1 ([5, p. 361, 14.2 Hilfssatz $])$. Assume $G$ is a group of order $p^{n}$ of maximal class. Then

(1) $\left|G / G^{\prime}\right|=p^{2}, G^{\prime}=\Phi(G)$ and $d(G)=2$;

(2) $\left|G_{i} / G_{i+1}\right|=p, i=2,3, \ldots, n-1$;

(3) for $i \geq 2, G_{i}$ is the unique normal subgroup of order $p^{n-i}$ of $G$;

(4) if $N \unlhd G,|G / N| \geq p^{2}$, then $G / N$ is also a p-group of maximal class;

(5) for $0 \leq i \leq n-1, Z_{i}(G)=G_{n-i}$;

(6) assume $p>2$. If $n>3$, then there does not exist any cyclic normal subgroup of order $p^{2}$.

Lemma 2.2 ([8]). Assume $G$ is a minimal nonabelian p-group. Then $G$ is one of the following groups:

(1) $Q_{8}$; 
(2) $M_{p}(n, m)=\left\langle a, b \mid a^{p^{n}}=b^{p^{m}}=1, a^{b}=a^{1+p^{n-1}}\right\rangle, n \geq 2, m \geq 1$; (metacyclic)

(3) $M_{p}(n, m, 1)=\langle a, b, c| a^{p^{n}}=b^{p^{m}}=c^{p}=1,[a, b]=c,[c, a]=[c, b]=$ $1\rangle, n \geq m$. If $p=2, m+n \geq 3$ (non-metacyclic).

Lemma $2.3([6])$. Assume $G$ is a finite p-group. If $G / N \cong M_{p}(n, m)$, where $N \leq Z(G)$ and $|N|=p$, then $G$ is one of the following mutually non-isomorphic groups:

I. $\left|G^{\prime}\right|=p$

(1) minimal nonabelian p-groups;

(2) direct product of a minimal nonabelian $p$-group and $C_{p}$;

II. $\left|G^{\prime}\right|=p^{2}$

$c(G)=2$

(1) $\left\langle a, b \mid a^{p^{n+1}}=b^{p^{m}}=1,[a, b]=a^{p^{n-1}}\right\rangle, n \geq 3, m \geq 2$;

(2) $\left\langle a, b \mid a^{p^{n+1}}=1, b^{p^{m}}=a^{p^{n}},[a, b]=a^{p^{n-1}}\right\rangle, m>n \geq 3$;

$c(G)=3$

(3) $\left\langle a, b \mid a^{8}=b^{2^{m}}=1,[a, b]=a^{2}\right\rangle$;

(4) $\left\langle a, b \mid a^{8}=b^{2^{m}}=1,[a, b]=a^{-2}\right\rangle$;

(5) $\left\langle a, b \mid a^{8}=1, b^{2^{m}}=a^{4},[a, b]=a^{-2}\right\rangle$;

(6) $\left\langle a, b \mid a^{p^{3}}=b^{p^{m}}=1,[a, b]=a^{p}\right\rangle, p \geq 3, m \geq 2$;

(7) $\left\langle a, b \mid a^{p^{3}}=1, b^{p^{m}}=a^{p^{2}},[a, b]=a^{p}\right\rangle, p \geq 3, m \geq 3$.

Lemma 2.4 ([10]). $Q_{8} * Q_{8} \cong D_{8} * D_{8}$.

Lemma 2.5 ([10, p. 51, 2.5.5]). Assume $G$ is a nonabelian 2-group. If $\mid G$ : $G^{\prime} \mid=4$, then $G$ is of maximal class.

Lemma 2.6 ([9]). Assume $G$ is a metaabelian group, $a, b \in G$ and $m, n$ are positive integers. Then

$$
\begin{gathered}
{\left[a^{m}, b^{n}\right]=\prod_{i=1}^{m} \prod_{j=1}^{n}[i a, j b]^{\left(\begin{array}{c}
m \\
i
\end{array}\right)\left(\begin{array}{c}
n \\
j
\end{array}\right),}} \\
\left(a b^{-1}\right)^{m}=a^{m}\left(\prod_{i+j \leq m}[i a, j b]^{\left(\begin{array}{c}
m \\
i+j
\end{array}\right)}\right) b^{-m},
\end{gathered}
$$

where $i, j$ are integers and satisfy $i+j \leq m$.

Lemma 2.7 ([2, p. 73, Lemma 4.2]). Assume $G$ is a p-group and $\left|G^{\prime}\right|=p$. Then $G=\left(A_{1} * A_{2} * \cdots * A_{s}\right) Z(G)$, where $A_{1}, A_{2}, \ldots, A_{s}$ are minimal nonabelian p-groups.

Lemma 2.8 ([7, p. 370, Lemma 3.2]). Assume $G$ is a finite p-group of order $\geq p^{3}, m>1$ and $p>2$. Then all nonnormal subgroups of $G$ are of order $p^{m}$ if and only if $G \cong M_{p}(n, m)$, where $m \leq n$. 
Lemma $2.9([7])$. Assume $G$ is a finite non-Dedekind p-group. Then all nonnormal subgroups of $G$ are of order $p$ if and only if $G$ is one of the following groups:

(1) $M_{p}(m, 1)$;

(2) $M_{p}(1,1,1) * C_{p^{n}}$;

(3) $D_{8} * Q_{8}$.

Lemma 2.10 ([15, Lemma 2.4]). Assume $E$ is a minimal nonabelian subgroup of a finite p-group. If $[G, E]=E^{\prime}$, then $G=E * C_{G}(E)$.

Lemma 2.11 ([15, Proposition 2.5]). Assume $G$ is a finite p-group and $\left|G^{\prime}\right|=$ p. If $H \leq G$ and $H \not \leq Z(G)$, then $H \unlhd G$ if and only if $G^{\prime} \leq H$.

Lemma 2.12. Assume $G$ is a finite non-Dedekind group. If every nonnormal subgroup $H$ of $G$ satisfies $\left|N_{G}(H): H\right|=p_{1} p_{2} \cdots p_{s}$, where $p_{i}$ is a prime and $s$ is a positive integer, then $p_{1}=p_{2}=\cdots=p_{s}=p$. That is, $G$ is a $p$-group.

Proof. By hypothesis we have $G$ is nilpotent. Since $G$ is non-Dedekind, there exists $P_{i} \in \operatorname{Syl}_{p_{i}}(G)$ such that $P_{i}$ is non-Dedekind. Assume $G=P_{i} \times K$. Since $P_{i}$ is non-Dedekind, there exists $H<P_{i}$ and $H \nsubseteq P_{i}$. Moreover, $H \nsubseteq G$. Since $(|H|,|K|)=1, N_{G}(H)=N_{P_{i}}(H) \times K$. It follows that $\left|N_{G}(H): H\right|=$ $\left|N_{P_{i}}(H): H\right||K|=p_{i}{ }^{x_{i}}|K|$. Since $H \times K \not G, N_{G}(H \times K)=N_{G}(H)$. It follows that $\left|N_{G}(H \times K): H \times K\right|=\left|N_{P_{i}}(H): H\right|=p_{i}{ }^{x_{i}}$. By hypothesis again, we have $K=1$. Thus $G=P_{i}$. Assume $p=p_{i}$. Then $G$ is a $p$-group.

Lemma 2.13. (1) If $G \in \mathcal{S}_{1}$, then $H \in \mathcal{S}_{1}$ for $H \leq G$.

(2) If $G \in \mathcal{S}_{i}$, then $G / N \in \mathcal{S}_{i}$ for $N \unlhd G$, where $i \geq 1$

Proof. (1) Assume $H \leq G$ and $K \leq H$. If $K \unlhd H$, then $K \nsupseteq G$. By hypothesis, $\left|N_{G}(K): K\right|=p$. Since $\left|N_{H}(K): K\right| \leq\left|N_{G}(K): K\right|$ and $K<N_{H}(K)$, $\left|N_{H}(K): K\right|=p$. So $H \in \mathcal{S}_{1}$.

(2) Assume $N \unlhd G$ and $H / N \Varangle G / N$. Then $H \not g G$. It follows that $\left|N_{G / N}(H / N): H / N\right|=\left|N_{G}(H) / N: H / N\right|=\left|N_{G}(H): H\right|=p^{i}$. Thus $G / N \in \mathcal{S}_{i}$.

\section{Classifying $\mathcal{S}_{1}$}

Lemma 3.1. A finite group $G$ is a Dedekind group if and only if all cyclic subgroups of $G$ are normal.

Lemma 3.2. A subgroup $N$ of a finite group $G$ is fully-normal if and only if all cyclic subgroups of $N$ are normal in $G$.

Lemma 3.3. Assume $G$ is a finite p-group. If $|G| \leq p^{3}$, then $G \in \mathcal{S}_{1}$.

Lemma 3.4. Assume $G$ is a minimal nonabelian $p$-group and $|G| \geq p^{4}$. If $G \in \mathcal{S}_{1}$, then $G \cong M_{p}(2,2)$. 
Proof. By Lemma $2.2, G \cong M_{p}(n, m, 1)$ or $G \cong M_{p}(n, m)$. If $G \cong M_{p}(n, m, 1)$, then by hypothesis we have $n+m+1 \geq 4$. From $n \geq m$ we get $n \geq 2$. Let $G=\left\langle a, b, c \mid a^{p^{n}}=b^{p^{m}}=c^{p}=1,[a, b]=c,[a, c]=[b, c]=1\right\rangle$. Obviously, $\langle b\rangle \not G$. On the other hand, $N_{G}(\langle b\rangle) \geq\left\langle a^{p}\right\rangle \times\langle c\rangle \times\langle b\rangle$. Thus $\mid N_{G}(\langle b\rangle)$ : $\langle b\rangle \mid \geq p^{n} \geq p^{2}$, a contradiction. Assume $G \cong M_{p}(n, m)$. If $n \geq 3$, then let $G=\left\langle a, b \mid a^{p^{n}}=b^{p^{m}}=1,[a, b]=a^{p^{n-1}}\right\rangle$. Obviously, $\langle b\rangle \not G$. On the other hand, $N_{G}(\langle b\rangle) \geq\left\langle a^{p}\right\rangle \times\langle b\rangle$. Thus $\left|N_{G}(\langle b\rangle):\langle b\rangle\right| \geq p^{n-1} \geq p^{2}$, a contradiction. So $n=2$. If $m \geq 3$, then let $G=\langle a, b| a^{p^{2}}=b^{p^{m}}=1,[a, b]=$ $\left.a^{p}\right\rangle$, where $\left\langle a b^{p^{m-2}}\right\rangle \not G, o\left(\left\langle a b^{p^{m-2}}\right\rangle\right)=p^{2}$. But $N_{G}\left(\left\langle a b^{p^{m-2}}\right\rangle\right)=\langle a\rangle \times\left\langle b^{p}\right\rangle$. So $\left|N_{G}\left(\left\langle a b^{p^{m-2}}\right\rangle\right):\left\langle a b^{p^{m-2}}\right\rangle\right| \geq p^{m-1} \geq p^{2}$, a contradiction. So $m=2$. It follows that $G \cong M_{p}(2,2)$.

Theorem 3.5. Assume $G$ is a finite p-group, $p>2$ and $|G| \geq p^{4}$. Then $G \in \mathcal{S}_{1}$ if and only if $G$ is abelian or $G \cong M_{p}(2,2)$.

Proof. $\Longleftarrow$ : If $G$ is abelian, the conclusion is true. Assume $G \cong M_{p}(2,2)$. Since $\Omega_{1}(G)=Z(G)$, all subgroups of order $p$ are normal. Obviously, all subgroups of order $p^{3}$ are normal. So if $H \not G$, then $|H|=p^{2}$. It follows that $H<N_{G}(H)<G$. Thus $\left|N_{G}(H): H\right|=p$. That is, $G \in \mathcal{S}_{1}$.

$\Longrightarrow$ : We use induction on $|G|$. If $|G|=p^{4}$ and $G \in \mathcal{S}_{1}$, then we can prove $G$ is abelian or $G \cong M_{p}(2,2)$. The conclusion is true. Assume the conclusion is true for groups of order $<|G|$. Since $G$ is a $p$-group, there exists $N \leq G^{\prime} \cap Z(G)$ and $|N|=p$. By Lemma 2.13 and $|G / N|<|G|$, we have, by induction hypothesis, $G / N$ is abelian or $G / N \cong M_{p}(2,2)$.

If $G / N \cong M_{p}(2,2)$, then $\left|(G / N)^{\prime}\right|=\left|G^{\prime} N / N\right|=\left|G^{\prime} / G^{\prime} \cap N\right|=\left|G^{\prime} / N\right|=p$. Thus $\left|G^{\prime}\right|=p^{2}$. By Lemma $2.3, G \cong\left\langle a, b \mid a^{p^{3}}=b^{p^{2}}=1,[a, b]=a^{p}\right\rangle$. Thus $\left\langle b^{p}\right\rangle \not G,\left|\left\langle b^{p}\right\rangle\right|=p$. But $N_{G}\left(\left\langle b^{p}\right\rangle\right)=\left\langle a^{p}, b\right\rangle \cong M_{p}(2,2)$. Thus $\mid N_{G}\left(\left\langle b^{p}\right\rangle\right)$ : $\left\langle b^{p}\right\rangle \mid=p^{3}$, a contradiction.

If $G / N$ is abelian, then $G$ is abelian or nonabelian. If $G$ is nonabelian, then $\left|(G / N)^{\prime}\right|=\left|G^{\prime} N / N\right|=\left|G^{\prime} / G^{\prime} \cap N\right|=\left|G^{\prime} / N\right|=1$. So $\left|G^{\prime}\right|=p$. By Lemma 2.7, $G \cong A_{1} * A_{2} * \cdots * A_{s} Z(G)$, where $A_{i}$ is minimal nonabelian. Assume $G=A_{1} * K$ without loss of generality. If $K \not \leq A_{1}$, then there exists $g \in K \backslash A_{1}$ such that $N_{G}(H) \geq\left\langle N_{A_{1}}(H), g\right\rangle$ for any $H \not A_{1}$. Thus $\left|N_{G}(H): H\right| \geq p^{2}$, a contradiction. It follows that $K \leq A_{1}$. That is, $G=A_{1}$. By Lemma 3.4 we get $G \cong M_{p}(2,2)$.

Lemma 3.6. Assume $G$ is a 2-group of maximal class. Then

(i) $G / Z(G) \cong D_{2^{n-1}}$;

(ii) every maximal subgroup of $G$ is cyclic or of maximal class.

Proof. By [5, Chapter III, 11.9b Satz], $G$ is isomorphic to one of the following: $D_{2^{n}}, Q_{2^{n}}$ or $S D_{2^{n}}$. It is straightforward by a simple calculation.

Theorem 3.7. Assume $G$ is a group of order $2^{n}$. Then $G \in \mathcal{S}_{1}$ if and only if $G$ is one of the following mutually non-isomorphism groups 

I. Dedekind 2-groups;
II. 2-groups of maximal class;
III. $\left\langle a, b \mid a^{2^{n-2}}=b^{4}=1,[a, b]=a^{-2}\right\rangle$;
IV. $\left\langle a, b \mid a^{2^{n-2}}=b^{4}=1,[a, b]=a^{-2+2^{n-3}}\right\rangle$.

Proof. $\Longleftarrow$ : If $G$ is a 2-group of maximal class, we prove $G \in \mathcal{S}_{1}$.

Assume $H \nless G$. If $|G: H|=2^{2}$, then $\left|N_{G}(H): H\right|=2$. Assume $|G: H| \geq$ $2^{3}$ without loss of generality.

Assume $G$ is a counterexample of the smallest order. Then there exists $H \nsupseteq G$ such that $\left|N_{G}(H): H\right|>2$. Since $N_{G}(H)<G$, there exists $M \lessdot G$ such that $N_{G}(H) \leq M$. By Lemma 3.6 we get $M$ is cyclic or a 2-group of maximal class. If $M$ is cyclic, then $H \unlhd G$, a contradiction. So $M$ is a 2-group of maximal class. If $H \unlhd M$, then $|M: H| \geq 2^{2}$ by $|G: H| \geq 2^{3}$. By Lemma 2.1 there exists $i$ such that $H=M_{i}$. Thus $H$ char $M \unlhd G$. It follows that $H \unlhd G$, a contradiction. Thus $H \nsubseteq M$. Since $G$ is a counterexample of the smallest order, $\left|N_{M}(H): H\right|=2$. On the other hand, $N_{M}(H)=N_{G}(H) \cap M=N_{G}(H)$. So $\left|N_{G}(H): H\right|=2$, a contradiction again. So the counterexample does not exist.

If $G$ is the group of type III, then $G /\left\langle b^{2}\right\rangle=\langle\bar{a}, \bar{b}| \bar{a}^{2^{n-2}}=\bar{b}^{2}=1,[\bar{a}, \bar{b}]=$ $\left.\bar{a}^{(-2)}\right\rangle \cong D_{2^{n-1}}$. Let $H \nsubseteq G$. Then $\left\langle b^{2}\right\rangle \leq H$. If not, let $K=\left\langle a, b^{2}\right\rangle$. Then $H \leq K$. In fact, $H \leq K \Longleftrightarrow G-H \supseteq G-K \Longleftrightarrow \forall g \in G-K \Longrightarrow$ $g \in G-H \Longleftrightarrow \forall g \in G-K \Longrightarrow g \notin H$. Since every element of $G$ has the form $b^{j} a^{i}, j=0,1,2,3$, we need to prove $b a^{i}, b^{-1} a^{i} \notin H$. By calculation, we have $\left(b a^{i}\right)^{2}=b a^{i} b a^{i}=b^{2}\left(a^{b}\right)^{i} a^{i}=b^{2}$ for any $i$. So $b a^{i} \notin H$. In the same way, $\left(b^{-1} a^{i}\right)^{2}=b^{-1} a^{i} b^{-1} a^{i}=b^{3} a^{i} b^{3} a^{i}=b a^{i} b a^{i}=b^{2}$. So $b^{-1} a^{i} \notin H$. Thus $H \leq K=\left\langle a, b^{2}\right\rangle$. Moreover, $\left\langle a, b^{2}\right\rangle$ is a fully-normal subgroup of $G$. In fact, let $g=a^{i} b^{2 j}$ for any $g \in\left\langle a, b^{2}\right\rangle$. Then $g^{a}=\left(a^{i} b^{2 j}\right)^{a}=g, g^{b}=\left(a^{i} b^{2 j}\right)^{b}=$ $\left(a^{b}\right)^{i} b^{2 j}=a^{-i} b^{2 j}=g^{-1}$. So $\langle g\rangle \unlhd G$. By Lemma $3.2,\left\langle a, b^{2}\right\rangle$ is a fully-normal subgroup of $G$. It follows that $H \unlhd G$, a contradiction. It follows that $\left\langle b^{2}\right\rangle \leq H$.

Since $H \unlhd G, \bar{H} \nsupseteq \bar{G}$. Since $\bar{G}$ is of maximal class, $\left|N_{\bar{G}}(\bar{H}): \bar{H}\right|=2$. Thus $\left|N_{G}(H): H\right|=\left|N_{\bar{G}}(\bar{H}): \bar{H}\right|=2$. That is, the group of type III is in $\mathcal{S}_{1}$.

If $G$ is the group of type IV, considering $G /\left\langle b^{2}\right\rangle$ and $G /\left\langle a^{2^{n-3}} b^{2}\right\rangle$, then

$$
\begin{aligned}
G /\left\langle b^{2}\right\rangle & =\left\langle\bar{a}, \bar{b} \mid \bar{a}^{2^{n-2}}=\bar{b}^{2}=1,[\bar{a}, \bar{b}]=\bar{a}^{\left(-2+2^{n-3}\right)}\right\rangle \\
& \cong S D_{2^{n-1}} \\
G /\left\langle a^{2^{n-3}} b^{2}\right\rangle & =\left\langle\bar{a}, \bar{b} \mid \bar{a}^{2^{n-2}}=1, \bar{b}^{2}=\bar{a}^{2^{n-3}},[\bar{a}, \bar{b}]=\bar{a}^{\left(-2+2^{n-3}\right)}\right\rangle \\
& \cong S D_{2^{n-1}}
\end{aligned}
$$

Let $H \nsubseteq G$. We prove $\left\langle b^{2}\right\rangle \leq H$ or $\left\langle a^{2^{n-3}} b^{2}\right\rangle \leq H$ as follows. If not, then, letting $K=\left\langle a, b^{2}\right\rangle$, we can prove $H \leq K$. In fact, $H \leq K \Longleftrightarrow G-H \supseteq$ $G-K \Longleftrightarrow \forall g \in G-K \Longrightarrow g \in G-H \Longleftrightarrow \forall g \in G-K \Longrightarrow g \notin H$. Since every element of $G$ has the form $b^{j} a^{i}, j=0,1,2,3$, we need to prove $b a^{i}, b^{-1} a^{i} \notin$ $H$. By calculation, we have $\left(b a^{i}\right)^{2}=b a^{i} b a^{i}=b^{2}\left(a^{b}\right)^{i} a^{i}=b^{2} a^{\left(-1+2^{n-3}\right) i} a^{i}=$ 
$b^{2} a^{2^{n-3} i}$ for any $i$. If $(i, 2)=1$, then $\left(b a^{i}\right)^{2}=b^{2} a^{2^{n-3}}$; if $2 \mid i$, then $\left(b a^{i}\right)^{2}=b^{2}$. So $b a^{i} \notin H$. In the same way, $\left(b^{-1} a^{i}\right)^{2}=b^{-1} a^{i} b^{-1} a^{i}=b^{3} a^{i} b^{3} a^{i}=b a^{i} b a^{i}$. So $b^{-1} a^{i} \notin H$. Thus $H \leq K=\left\langle a, b^{2}\right\rangle$. Moreover, $\left\langle a, b^{2}\right\rangle$ is a fully-normal subgroup of $G$. In fact, let $g=a^{i} b^{2 j}$ for any $g \in\left\langle a, b^{2}\right\rangle$. Then $g^{a}=\left(a^{i} b^{2 j}\right)^{a}=g, g^{b}=$ $\left(a^{i} b^{2 j}\right)^{b}=\left(a^{b}\right)^{i} b^{2 j}=a^{-i+2^{n-3}} b^{2 j}$. If $(i, 2)=1, g^{b}=a^{-i+2^{n-3}} b^{2 j}=g^{-1+2^{n-3}}$; if $2 \mid i, g^{b}=a^{-i} b^{2 j}=g^{-1}$. Thus $\langle g\rangle \unlhd G$. By Theorem 3.2, we get $\left\langle a, b^{2}\right\rangle$ is a fully-normal subgroup of $G$. Thus $H \unlhd G$, a contradiction. It follows that $\left\langle b^{2}\right\rangle \leq H$ or $\left\langle a^{2^{n-3}} b^{2}\right\rangle \leq H$.

Since $H \unlhd G, \bar{H} \sharp \bar{G}$. Since $\bar{G}$ is of maximal class, $\left|N_{\bar{G}}(\bar{H}): \bar{H}\right|=2$. Thus $\left|N_{G}(H): H\right|=\left|N_{\bar{G}}(\bar{H}): \bar{H}\right|=2$. That is, the group of type IV is in $\mathcal{S}_{1}$.

$\Longrightarrow$ : Case 1: $d(G) \geq 3$.

Assume $G \in \mathcal{S}_{1}$ and $G$ is nonabelian. We prove $G$ is a nonabelian Dedekind 2-group as follows.

Assume $G$ is a counterexample of the smallest order. Since $\Phi(G) \neq 1$, we can take $N \leq \Phi(G)$ such that $|N|=2$ and $N \unlhd G$. Thus $d(G / N)=$ $d(G) \geq 3$. By Lemma 2.13, $G / N$ is a nonabelian Dedekind 2-group. Since $G$ is a counterexample of the smallest order, by Lemma 3.1 there exists $a \in G$ such that $\langle a\rangle \nexists G$. So $N \cap\langle a\rangle=1$. Since $N_{G}(\langle a\rangle) \geq\langle N, a\rangle=N \times\langle a\rangle$, $\left|N_{G}(\langle a\rangle):\langle a\rangle\right|=2$ by hypothesis. So $N_{G}(\langle a\rangle)=N \times\langle a\rangle$. Since $G / N$ is a Dedekind 2-group, $(N \times\langle a\rangle) /\langle a\rangle \unlhd G / N$. Thus $N \times\langle a\rangle \unlhd G$.

We calculate $\left|\left\{\langle a\rangle^{g} \mid g \in G\right\}\right|$ as follows. First, we prove $\left|\left\{\langle a\rangle^{g} \mid g \in G\right\}\right|=$ $\left|G: N_{G}(\langle a\rangle)\right| \geq 2^{2}$. Since $d(G) \geq 3,|G /\langle a, \Phi(G)\rangle| \geq 2^{2}$. Let $\bar{G}=G / N_{G}(\langle a\rangle)$. Then $\Phi(\bar{G})=\Phi\left(G / N_{G}(\langle a\rangle)\right)=\left(\Phi(G) N_{G}(\langle a\rangle)\right) / N_{G}(\langle a\rangle)$, and $\Phi(G) N_{G}(\langle a\rangle)=$ $\Phi(G)(N \times\langle a\rangle)=\Phi(G)\langle a\rangle=\langle a, \Phi(G)\rangle$. So $\bar{G} / \Phi(\bar{G}) \cong G /(\langle a, \Phi(G)\rangle)$. Since $|G:(\langle a, \Phi(G)\rangle)| \geq 2^{2},|\bar{G} / \Phi(\bar{G})| \geq 2^{2}$. That is, $d(\bar{G}) \geq 2$. Therefore $|\bar{G}| \geq 2^{2}$, that is, $\left|G: N_{G}(\langle a\rangle)\right| \geq 2^{2}$.

On the other hand, since $\langle a\rangle \leq N \times\langle a\rangle,\langle a\rangle^{g} \leq(N \times\langle a\rangle)^{g}=N \times\langle a\rangle$. So $\langle a\rangle^{g} \lessdot N \times\langle a\rangle$. Since $d(N \times\langle a\rangle)=2, N \times\langle a\rangle$ has three maximal subgroups. It follows that $\left|\left\{\langle a\rangle^{g} \mid g \in G\right\}\right| \leq 2$, a contradiction. So the counterexample does not exist.

Case $2: d(G) \leq 2$.

We use induction on $|G|$. If $|G|=2^{4}$ and $G \in \mathcal{S}_{1}$, then we can prove $G$ is a Dedekind group, a group of order $2^{4}$ of maximal class $2^{4}$ or $G \cong M_{2}(2,2)$. The conclusion is true. Assume the conclusion is true for groups of order $<|G|$. Since $G$ is a 2-group, there exists $N \leq G^{\prime} \cap Z(G)$ and $|N|=2$. Since the condition is inheritable by quotient groups and $|G / N|<|G|, G / N$ is one of the groups listed in theorem by induction hypothesis.

If $G / N$ is abelian, then, in the same way as that in the case $p>2$, we have $G$ is abelian or $G \cong Q_{8}, D_{8}$ or $M_{2}(2,2)$.

If $G / N$ is a 2-groups of maximal class, then $\left|(G / N)^{\prime}\right|=2^{n-2}$ by Lemma 2.1. Since $(G / N)^{\prime}=G^{\prime} N / N \cong G^{\prime} / G^{\prime} \cap N=G^{\prime} / N,\left|G^{\prime} / N\right|=2^{n-2}$. Thus $\left|G^{\prime}\right|=2^{n-1}$. It follows that $\left|G / G^{\prime}\right|=4$. By Lemma 2.5 we get $G$ is a 2 -group of maximal class. 
If $G / N$ is the group of type III. That is, $\bar{G} \cong\langle\bar{a}, \bar{b}| \bar{a}^{2^{n-2}}=\bar{b}^{4}=1,[\bar{a}, \bar{b}]=$ $\left.\bar{a}^{(-2)}\right\rangle$. Assume $N=\langle x\rangle$. Then $G=\langle a, b| a^{2^{n-2}}=x^{i}, b^{4}=x^{j},[a, b]=$ $\left.a^{-2} x^{k}, x^{2}=1,[x, a]=[x, b]=1\right\rangle$. Let $K=\left\langle a^{-2} x^{k}\right\rangle$. It is easy to prove $G / K$ is abelian. Thus $G^{\prime} \leq K$. But $K \leq G^{\prime}$. So $G^{\prime}=K$. That is, $G^{\prime}$ is cyclic. Since $\left|G^{\prime}\right|=2^{n-2}$, it follows by $[a, b]^{2^{n-2}}=1$ that $o(a)=2^{n-1}$ and $N=\left\langle a^{2^{n-2}}\right\rangle$. Thus we get the following groups:

(a1) $\left\langle a, b \mid a^{2^{n-1}}=1, b^{4}=1,[a, b]=a^{-2}\right\rangle$;

(a2) $\left\langle a, b \mid a^{2^{n-1}}=1, b^{4}=1,[a, b]=a^{-2+2^{n-2}}\right\rangle$;

(a3) $\left\langle a, b \mid a^{2^{n-1}}=1, b^{4}=a^{2^{n-2}},[a, b]=a^{-2}\right\rangle$;

(a4) $\left\langle a, b \mid a^{2^{n-1}}=1, b^{4}=a^{2^{n-2}},[a, b]=a^{-2+2^{n-2}}\right\rangle$;

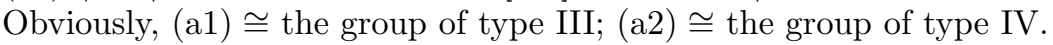

For (a3), let $H=\left\langle a^{2^{n-3}} b^{2}\right\rangle$. Then $|H|=2$ and $H \not G, N_{G}(H)=\left\langle a, b^{2}\right\rangle \lessdot G$. Thus $\left|N_{G}(H): H\right| \geq 2^{3}$, a contradiction. For (a4), let $a^{\prime}=a b^{2}, b^{\prime}=b$. Then $(\mathrm{a} 4) \cong(\mathrm{a} 3)$.

If $G / N \cong$ the group of type IV, then, by a similar argument as that case of above paragraph, no new groups occur. The theorem is proved.

\section{Classifying $\mathcal{S}_{2}$}

Lemma 4.1. Assume $G$ is a p-group. If $G \in \mathcal{S}_{2}$ and $|G: H| \leq p^{2}$ for $H \leq G$, then $H \unlhd G$. In particular, if $G$ is nonabelian, then $G \cong Q_{8}$ or $|G| \geq p^{4}$.

Proof. Assume $|G|=p^{n}$ and $|G: H|=p^{2}$. If $H \not G$, then $H<N_{G}(H)<G$. Thus $\left|N_{G}(H)\right|=p^{n-1}$. It follows that $\left|N_{G}(H): H\right|=p$, a contradiction. So $H \unlhd G$. In particular, if $G$ is nonabelian and $|G|=p^{3}$, then $G \cong Q_{8}$.

Lemma 4.2. Assume $G$ is a minimal nonabelian p-group and $|G| \geq p^{4}$. Then $G \in \mathcal{S}_{2}$ if and only if $G \cong M_{p}(3, m)$, where $m \leq 3$.

Proof. $\Longrightarrow$ : By Lemma 2.2, $G \cong M_{p}(n, m, 1)$ or $G \cong M_{p}(n, m)$.

If $G \cong M_{p}(n, m)=\left\langle a, b \mid a^{p^{n}}=1, b^{p^{m}}=1,[a, b]=a^{p^{n-1}}\right\rangle$, then, if $n \geq 4$, letting $H=\langle b\rangle$, we get $H \notin G$. But $N_{G}(H) \geq\left\langle a^{p}\right\rangle \times\langle b\rangle$. Thus $\mid N_{G}(H)$ : $H \mid \geq p^{n-1} \geq p^{3}$, a contradiction. If $n=2$, letting $H=\langle b\rangle$, we get $H \not \sharp G$. But $|G: H|=p^{2}$. This contradicts Lemma 4.1. If $G \cong M_{p}(3, m)$ and $m \geq 4$, letting $H=\left\langle a b^{p^{m-3}}\right\rangle$, we get $H \not G$. But $N_{G}(H) \geq\langle a\rangle \times\left\langle b^{p}\right\rangle$. Thus $\mid N_{G}(H)$ : $H \mid \geq p^{m-1} \geq p^{3}$, a contradiction. Thus $G \cong M_{p}(3, m)$, where $m \leq 3$.

If $G \cong M_{p}(n, m, 1)$, then, by a similar argument as that case of above paragraph, the case does not occur.

$\Longleftarrow$ : We check case by case.

If $G \cong M_{p}(3,3)$, then $Z(G)=\left\langle a^{p}\right\rangle \times\left\langle b^{p}\right\rangle, G^{\prime}=\left\langle a^{p^{2}}\right\rangle, \Omega_{1}(G)=\left\langle a^{p^{2}}\right\rangle \times$ $\left\langle b^{p^{2}}\right\rangle, \Omega_{2}(G)=\left\langle a^{p}\right\rangle \times\left\langle b^{p}\right\rangle$. If $|H|=p^{5}$, then $H \lessdot G$. Thus $H \unlhd G$. If $|H|=p^{4}$, then $|H \cap\langle a\rangle| \neq 1$. If not, $|H\langle a\rangle|>|G|$, a contradiction. It follows that $G^{\prime} \leq H$, and $H \unlhd G$. If $|H|=p^{3}$ and $H \not G$, then $|H \cap Z(G)| \leq p^{2}$, and $N_{G}(H) \geq\langle H, Z(G)\rangle$. Thus $\left|N_{G}(H)\right| \geq \frac{|H||Z(G)|}{|H \cap Z(G)|} \geq \frac{p^{3} \cdot p^{4}}{p^{2}}=p^{5}$. It follows that 
$\left|N_{G}(H)\right|=p^{5}$, and $\left|N_{G}(H): H\right|=p^{2}$. If $|H|=p^{2}$, then $H \leq \Omega_{2}(G) \leq Z(G)$. Thus $H \unlhd G$. If $|H|=p$, then $H \leq \Omega_{1}(G) \leq Z(G)$. Thus $H \unlhd G$. So $G \in \mathcal{S}_{2}$.

If $G \cong M_{p}(3,2)$ or $G \cong M_{p}(3,1)$, then $G \in \mathcal{S}_{2}$ by a similar argument as that case of above paragraph.

Lemma 4.3. Assume $G$ is a non-Dedekind p-group and $|G| \geq p^{4}, K$ is a minimal nonabelian p-group. If $G \cong K \times C_{p}$, then $G \notin \mathcal{S}_{2}$.

Proof. By Lemma $2.2, K \cong M_{p}(n, m)$ or $M_{p}(n, m, 1)$. If $K \cong M_{p}(n, m, 1)$, then $G \cong K \times N$, where $N \cong C_{p}$, and $G / N \cong M_{p}(n, m, 1)$. By Lemma 2.13 and Lemma $4.2, G \notin \mathcal{S}_{2}$. If $K \cong M_{p}(n, m)$, then $G=\langle a, b, c| a^{p^{n}}=1, b^{p^{m}}=$ $\left.1, c^{p}=1,[a, b]=a^{p^{n-1}},[a, c]=[b, c]=1\right\rangle$. If $n \geq 3$, then, by letting $H=\langle b\rangle$, we get $H \not G$. But $N_{G}(H)=\left\langle a^{p}\right\rangle \times\langle b\rangle \times\langle c\rangle$. Thus $\left|N_{G}(H): H\right| \geq p^{n} \geq p^{3}$, a contradiction. If $n=2$, let $H \cong\langle b, c\rangle$. Then $H \nsubseteq G$. But $|G: H|=p^{2}$. This contradicts Lemma 4.1. So $G \notin \mathcal{S}_{2}$.

Theorem 4.4. Assume $G$ is a finite p-groups. Then $G \in \mathcal{S}_{2}$ if and only if $G$ is one of the following mutually non-isomorphic groups

(1) $M_{p}(3, m)$, where $m \leq 3$;

(2) $M_{p}(1,1,1) * C_{p^{2}}$

(3) $\left\langle a, b, c \mid a^{p^{2}}=b^{p^{2}}=c^{p}=1,[a, b]=1,[a, c]=b^{k p},[b, c]=a^{p} b^{h p}\right\rangle$, if $p>2, k+4^{-1} h^{2}$ is a fixed quadratic non-residue $(\bmod p)$, where $k=1$ or $\nu, \nu$ is a fixed quadratic non-residue $(\bmod p), h=0,1, \ldots, \frac{p-1}{2}$. If $p=2$, then $k=1, h=1$;

(4) $Q_{8} \times C_{4}$;

(5) $\left\langle a, b, c \mid a^{4}=b^{4}=1, c^{2}=b^{2},[a, b]=1,[a, c]=b^{2},[b, c]=a^{2}\right\rangle$;

(6) $\langle a, b, c| a^{4}=b^{4}=c^{4}=1,[a, b]=c^{2},[a, c]=b^{2} c^{2},[b, c]=a^{2} b^{2},\left[c^{2}, a\right]=$ $\left.\left[c^{2}, b\right]=1\right\rangle$

(7) Dedekind groups.

Proof. $\Longrightarrow$ : We use induction on $|G|$. If $|G|=p^{4}$ and $G \in \mathcal{S}_{2}$, then we can prove $G$ is a Dedekind group, or $G \cong M_{p}(3,1)$ or $G \cong M_{p}(1,1,1) * C_{p^{2}}$. The conclusion is true. Assume the conclusion is true for groups of order $<|G|$. Since $G$ is a $p$-group, there exists $N \leq G^{\prime} \cap Z(G)$ and $|N|=p$. Since the condition is inheritable by quotient groups and $|G / N|<|G|, G / N$ is the group listed in Theorem by induction hypothesis.

Case 1: If $G / N \cong M_{p}(3, m)=\left\langle\bar{a}, \bar{b} \mid \bar{a}^{p^{3}}=1, \bar{b}^{p^{m}}=1,[\bar{a}, \bar{b}]=\bar{a}^{p^{2}}\right\rangle$, then, by $\left|(G / N)^{\prime}\right|=\left|G^{\prime} N / N\right|=\left|G^{\prime} / G^{\prime} \cap N\right|=\left|G^{\prime} / N\right|=p$, we have $\left|G^{\prime}\right|=p^{2}$. By Lemma $2.3, G \cong\left\langle a, b \mid a^{p^{4}}=1, b^{p^{m}}=1,[a, b]=a^{p^{2}}\right\rangle$, where $m=2,3$. Let $H=\left\langle b^{p}\right\rangle$. Then $H \not G$. But $N_{G}(H) \geq\left\langle a^{p^{2}}, b\right\rangle$. So $\left|N_{G}(H): H\right| \geq p^{3}$, a contradiction.

Case 2: If $G / N \cong M_{p}(1,1,1) * C_{p^{2}}=\langle\bar{a}, \bar{b}, \bar{c}\rangle=\left\langle\bar{a}^{p^{2}}=1, \bar{b}^{p}=1, \bar{c}^{p}=\right.$ $\left.1,[\bar{b}, \bar{c}]=\bar{a}^{p},[\bar{a}, \bar{b}]=1,[\bar{a}, \bar{c}]=1\right\rangle$. Let $N=\langle x\rangle$. Then $G=\langle a, b, c| a^{p^{2}}=$ $x^{i}, b^{p}=x^{j}, c^{p}=x^{k},[a, b]=x^{l},[a, c]=x^{m},[b, c]=a^{p} x^{n}, x^{p}=1,[x, a]=[x, b]=$ $[x, c]=1\rangle$. 
It is easy to prove the following facts:

1. $o(a)=p^{2}$;

2. $G^{\prime} \cong C_{p} \times C_{p}$ and $c(G)=2$;

3. $\mho_{1}(G) \leq Z(G)$;

4. $j, k$ are not zero in the same time.

We discuss in two cases: (i) $b^{p} \neq 1$ and $c^{p}=1$, (ii) $b^{p} \neq 1$ and $c^{p} \neq 1$.

(i) $b^{p} \neq 1$ and $c^{p}=1$.

Then $G=\langle a, b, c| a^{p^{2}}=1, b^{p}=x^{j}, c^{p}=1,[a, b]=x^{l},[a, c]=x^{m},[b, c]=$ $\left.a^{p} x^{n}, x^{p}=1,[x, a]=[x, b]=[x, c]=1\right\rangle$. Let $H=\langle a, c\rangle$. If $[a, c]=1$, then $H \not G$, and $|G: H|=p^{2}$. This contradicts Lemma 4.1. Thus $m \not \equiv 0(\bmod p)$.

(ia) $p>2$.

If $[a, b] \neq 1$, then, by letting $b_{1}=b c^{t}$ satisfying $l+m t \equiv 0(\bmod p)$, it reduces to the case $[a, b]=1$. Assume $G=\langle a, b, c| a^{p^{2}}=1, b^{p}=x, c^{p}=1,[a, b]=$ $\left.1,[a, c]=x^{m},[b, c]=a^{p} x^{n}, x^{p}=1,[x, a]=[x, b]=[x, c]=1\right\rangle \cong\langle a, b, c| a^{p^{2}}=$ $\left.1, b^{p^{2}}=1, c^{p}=1,[a, b]=1,[a, c]=b^{m p},[b, c]=a^{p} b^{n p}\right\rangle$.

If $m \equiv s^{2}(\bmod p)$, then, replacing $a$ by $a^{s^{-1}}$, and $c$ by $c^{s^{-1}}$, and letting $h=n s^{-1}$, we have $G=\langle a, b, c| a^{p^{2}}=1, b^{p^{2}}=1, c^{p}=1,[a, b]=1,[a, c]=$ $\left.b^{p},[b, c]=a^{p} b^{h p}\right\rangle$. Replacing $a$ by $a^{-1}$, and $c$ by $c^{-1}$, we have $h \leq \frac{p-1}{2}$. Let $H=\left\langle a b^{i}, c\right\rangle$. If $h i+1 \equiv i^{2}(\bmod p)$, in other words, $1+\frac{h^{2}}{4}$ is a quadratic residue $(\bmod p)$, then $H \cong M_{p}(2,1)$, and $|H|=p^{3}$. Obviously, $H \nsubseteq G$. But $|G: H|=p^{2}$. This contradicts Lemma 4.1. So $1+\frac{h^{2}}{4}$ is a quadratic nonresidue $(\bmod p)$.

If $m \equiv \nu s^{2}(\bmod p)$, where $\nu \neq 0$, then, replacing $a$ by $a^{s^{-1}}, c$ by $c^{s^{-1}}$ and letting $h=n s^{-1}, G=\langle a, b, c| a^{p^{2}}=1, b^{p^{2}}=1, c^{p}=1,[a, b]=1,[a, c]=$ $\left.b^{\nu p},[b, c]=a^{p} b^{h p}\right\rangle$. Again replacing $a$ by $a^{-1}$ and $c$ by $c^{-1}$, we have $h \leq \frac{p-1}{2}$. Let $H=\left\langle a b^{i}, c\right\rangle$. If $h i+\nu \equiv i^{2}(\bmod p)$, in other words, $\nu+\frac{h^{2}}{4}$ is a quadratic residue $(\bmod p)$, then $H \cong M_{p}(2,1)$, and $|H|=p^{3}$. Obviously, $H \not g G$, But $|G: H|=p^{2}$. This contradicts Lemma 4.1. So $\nu+\frac{h^{2}}{4}$ is a quadratic nonresidue $(\bmod p)$.

So, if $p>2$ and $G / N \cong M_{p}(1,1,1) * C_{p^{2}}$, then $G$ is the group of type (3).

(ib) $p=2$.

Assume $G=\langle a, b, c| a^{4}=1, b^{2}=x, c^{2}=1,[b, c]=a^{2} x^{n},[a, b]=x^{l},[a, c]=$ $\left.x, x^{2}=1,[x, a]=[x, b]=[x, c]=1\right\rangle$. If $l=n$, then, by letting $H=\langle a b, c\rangle$, we have $H \unlhd G$. But $|G: H|=p^{2}$. This contradicts Lemma 4.1. So $l \neq n$. Thus we get two groups:

$$
\begin{aligned}
G_{(11)}= & \langle a, b, c| a^{4}=1, b^{2}=x, c^{2}=1,[b, c]=a^{2},[a, b]=x,[a, c]=x, x^{2}=1, \\
& {[x, a]=[x, b]=[x, c]=1\rangle } \\
\cong & \left\langle a, b, c \mid a^{4}=1, b^{4}=1, c^{2}=1,[b, c]=a^{2},[a, b]=b^{2},[a, c]=b^{2}\right\rangle, \\
G_{(12)}= & \left\langle a_{1}, b_{1}, c_{1}\right| a_{1}^{4}=1, b_{1}^{2}=x_{1}, c_{1}^{2}=1,\left[b_{1}, c_{1}\right]=a_{1}{ }^{2} x_{1},\left[a_{1}, b_{1}\right]=1, \\
& {\left.\left[a_{1}, c_{1}\right]=x_{1}, x_{1}{ }^{2}=1,\left[x_{1}, a_{1}\right]=\left[x_{1}, b_{1}\right]=\left[x_{1}, c_{1}\right]=1\right\rangle }
\end{aligned}
$$




$$
\begin{aligned}
\cong & \left\langle a_{1}, b_{1}, c_{1}\right| a_{1}{ }^{4}=1, b_{1}{ }^{4}=1, c_{1}{ }^{2}=1,\left[b_{1}, c_{1}\right]=a_{1}{ }^{2} b_{1}{ }^{2},\left[a_{1}, b_{1}\right]=1, \\
& {\left.\left[a_{1}, c_{1}\right]=b_{1}{ }^{2}\right\rangle . }
\end{aligned}
$$

Let $\sigma: a_{1} \longrightarrow a, b_{1} \longrightarrow a b c, c_{1} \longrightarrow c$. Then $G_{(11)} \cong G_{(12)} \cong$ the group of type (3).

(ii) $b^{p} \neq 1$ and $c^{p} \neq 1$

If $p>2$, then, by letting $c_{1}=c b^{t}$ satisfying $j t+k \equiv 0(\bmod p)$, it reduces to the case (ia).

If $p=2$, assume $G=\langle a, b, c| a^{4}=1, b^{2}=x, c^{2}=x,[b, c]=a^{2} x^{n},[a, b]=$ $\left.x^{l},[a, c]=x^{m}, x^{2}=1,[x, a]=[x, b]=[x, c]=1\right\rangle$. Since $G^{\prime} \cong C_{2} \times C_{2}, l, m$ are not zero in the same time.

(ii-1) $m=0, l=1$.

If $n=0$, then $G \cong\langle a, b, c| a^{4}=1, b^{4}=1, c^{2}=b^{2},[b, c]=a^{2},[a, b]=$ $\left.b^{2},[a, c]=1\right\rangle$. Let $a_{1}=a, b_{1}=c, c_{1}=b$. Then $G \cong$ the group of type (5). If $n=1$, then $G \cong\langle a, b, c| a^{4}=1, b^{4}=1, c^{2}=b^{2},[b, c]=a^{2} b^{2},[a, b]=b^{2},[a, c]=$ $1\rangle$. Let $a_{1}=a, b_{1}=b, c_{1}=a b c$. Then $G \cong G_{(11)} \cong$ the group of type (3).

(ii-2) $m=1, l=0$.

Then $G=\langle a, b, c| a^{4}=1, b^{2}=x, c^{2}=x,[b, c]=a^{2} x^{n},[a, b]=1,[a, c]=$ $\left.x, x^{2}=1,[x, a]=[x, b]=[x, c]=1\right\rangle$. Let $a_{1}=a, b_{1}=c, c_{1}=b$. Then it reduces to the case (ii -1 ).

(ii-3): $m=1, l=1$.

Then $G=\langle a, b, c| a^{4}=1, b^{2}=x, c^{2}=x,[b, c]=a^{2} x^{n},[a, b]=x,[a, c]=$ $\left.x, x^{2}=1,[x, a]=[x, b]=[x, c]=1\right\rangle$. Let $a_{1}=a, b_{1}=b, c_{1}=a b c$. Then it reduces to the case (i) or (ii -1$)$.

Case 3: If $G / N \cong\langle\bar{a}, \bar{b}, \bar{c}| \bar{a}^{p^{2}}=1, \bar{b}^{p^{2}}=1, \bar{c}^{p}=1,[\bar{a}, \bar{b}]=\overline{1},[\bar{a}, \bar{c}]=$ $\left.\bar{b}^{k p},[\bar{b}, \bar{c}]=\bar{a}^{p} \bar{b}^{h p}\right\rangle$. Assume $N=\langle x\rangle$, then $G=\langle a, b, c| a^{p^{2}}=x^{i}, b^{p^{2}}=x^{j}, c^{p}=$ $x^{k},[a, b]=x^{l},[a, c]=b^{k p} x^{m},[b, c]=a^{p} b^{h p} x^{n}, x^{p}=1,[x, a]=[x, b]=[x, c]=$ $1\rangle$.

First, we prove the following facts:

1. $o(a)=p^{2}$ and $o(b)=p^{2}$;

2. $G^{\prime} \cong C_{p}^{3}$ and $c(G)=2$;

3. $c^{p} \neq 1$.

In fact, since $c(G / N)=2,(G / N)_{3}=G_{3} N / N=1$. Thus $G_{3} \leq N \leq Z(G)$. That is, $G_{4}=1$. Since $G^{\prime \prime} \leq G_{4}, G^{\prime}$ is abelian. Since $\left|(G / N)^{\prime}\right|=p^{2},\left|G^{\prime}\right|=p^{3}$. It follows by $c^{p} \in Z(G)$ that $\left[a, c^{p}\right]=1,\left[b, c^{p}\right]=1$.

By the formula of Lemma 2.6, we have

$$
\begin{aligned}
{\left[b^{k p}, c\right] } & =[b, c]^{k p}[b, c, b]^{\left(\begin{array}{c}
k p \\
2
\end{array}\right)}=\left(a^{p} b^{h p} x^{n}\right)^{k p}\left[a^{p} b^{h p} x^{n}, b\right] \\
& =a^{k p^{2}}\left[a^{p}, b^{-h p}\right] b^{h k p^{2}}\left[a^{p} b^{h p}, b\right] \\
& =a^{k p^{2}} b^{h k p^{2}}\left[a^{p}, b\right]^{b^{h p}}=a^{k p^{2}} b^{h k p^{2}} .
\end{aligned}
$$

It follows that

$$
1=\left[a, c^{p}\right]=[a, c]^{p}[a, c, c]^{\left(\begin{array}{c}
p \\
2
\end{array}\right)}=[a, c]^{p}\left[b^{k p}, c\right]^{\left(\begin{array}{l}
p \\
2
\end{array}\right)}=b^{k p^{2}}\left(a^{k p^{2}} b^{h k p^{2}}\right)^{\left(\begin{array}{c}
p \\
2
\end{array}\right)} .
$$




$$
\begin{aligned}
1 & =\left[b, c^{p}\right]=[b, c]^{p}[b, c, c]^{\left(\begin{array}{l}
p \\
2
\end{array}\right)}=\left(a^{p} b^{h p} x^{n}\right)^{p}\left[a^{p} b^{h p} x^{n}, c\right]^{\left(\begin{array}{c}
p \\
2
\end{array}\right)} \\
& =a^{p^{2}} b^{h p^{2}}\left(\left[a^{p}, c\right]^{b^{h p}}\left[b^{h p}, c\right]\right)^{\left(\begin{array}{l}
p \\
2
\end{array}\right)}=a^{p^{2}} b^{h p^{2}}\left(b^{k p^{2}} a^{h p^{2} b^{h^{2} p^{2}}}\right)^{\left(\begin{array}{c}
p \\
2
\end{array}\right) .}
\end{aligned}
$$

If $p>2$, then it follows from (1) and (2) that $\left[a, c^{p}\right]=b^{k p^{2}}=1,\left[b, c^{p}\right]=$ $a^{p^{2}} b^{h p^{2}}=1$. Thus $o(a)=p^{2}, o(b)=p^{2}$.

If $p=2$, then $h=k=1$. It follows from (1) and (2) that $\left[a, c^{2}\right]=a^{4}=1$, $\left[b, c^{2}\right]=b^{4}=1$. Thus $o(a)=4, o(b)=4$.

Since $\bar{G}^{\prime}=\overline{G^{\prime}}=\left\langle\overline{a^{p}}, \overline{b^{p}}\right\rangle, G^{\prime}=\left\langle a^{p}, b^{p}, x\right\rangle \cong C_{p}{ }^{3}$. So $l \neq 0$. Since $G^{\prime} \leq Z(G)$, $c(G)=2$. Moreover, assume $c^{p} \neq 1$. If not, let $H=\langle c\rangle$. Then $H \notin G$. But $N_{G}(H) \geq\left\langle a^{p}, b^{p}, c, x\right\rangle$. So $\left|N_{G}(H): H\right| \geq p^{3}$, a contradiction.

If $p>2$, assume $G=\langle a, b, c| a^{p^{2}}=1, b^{p^{2}}=1, c^{p}=x,[a, b]=x^{l},[a, c]=$ $\left.b^{k p} x^{m},[b, c]=a^{p} b^{h p} x^{n}, x^{p}=1,[x, a]=[x, b]=[x, c]=1\right\rangle$. Obviously, $|G|=p^{6}$. Since $c(G)=2, G$ is $p$-abelian. It follows that $\Omega_{1}(G)=\left\langle a^{p}\right\rangle \times\left\langle b^{p}\right\rangle \times\langle x\rangle \leq Z(G)$. We will prove $G$ does not satisfy the condition of theorem.

Assume $H \leq G$. If $|H|=p^{3}$, then $H$ is abelian. In fact, if $\exp (H)=p$, then $H=\Omega_{1}(\bar{G})$. Thus $H$ is abelian. If $\exp (H)=p^{2}$ and $H$ is not abelian, then $H \cong M_{p}(2,1)$. This contradicts $\Omega_{1}(G) \leq Z(G)$. If $|H|=p^{5}$, then $H \triangleleft G$. If $|H|=p^{4}$ and $H \unlhd G$, then $G$ is meta-Hamilton $p$-group. But by the classification of meta-Hamilton $p$-group [1], we know $G$ is not a meta-Hamilton $p$-group. Thus there exists a nonnormal subgroup $H$ of order $p^{4}$. It follows that $|G: H|=p^{2}$. But this contradicts Lemma 4.1. So $G$ does not satisfy the condition of theorem.

If $p=2$, assume $G=\langle a, b, c| a^{4}=1, b^{4}=1, c^{2}=x,[a, b]=x,[a, c]=$ $\left.b^{2} x^{m},[b, c]=a^{2} b^{2} x^{n}, x^{2}=1,[x, a]=[x, b]=[x, c]=1\right\rangle$, where $m, n=0,1$. If $m=n$, then, by letting $H=\langle a c, b\rangle \cong M_{2}(2,2)$, we have $|H|=2^{4}$. Obviously, $H \unlhd G$. But $|G: H|=2^{2}$. This contradicts Lemma 4.1. If $m=0, n=1$, then, by letting $H=\langle a b, b c\rangle \cong M_{2}(2,2)$, we have $|H|=2^{4}$. Obviously, $H \nsubseteq G$. But $|G: H|=p^{2}$. This contradicts Lemma 4.1 again. If $m=1, n=0$, Then $G \cong\langle a, b, c| a^{4}=1, b^{4}=1, c^{4}=1,[a, b]=c^{2},[a, c]=b^{2} c^{2},[b, c]=$ $\left.a^{2} b^{2},\left[c^{2}, a\right]=\left[c^{2}, b\right]=1\right\rangle \cong$ the group of type $(6)$.

Case 4: If $G / N \cong\langle\bar{a}, \bar{b}, \bar{c}| \bar{a}^{4}=1, \bar{b}^{2}=\bar{a}^{2}, \bar{c}^{4}=1,[\bar{a}, \bar{b}]=\bar{a}^{2},[\bar{a}, \bar{c}]=$ $\overline{1},[\bar{b}, \bar{c}]=\overline{1}\rangle$. Assume $N=\langle x\rangle$. Then $G=\langle a, b, c| a^{4}=x^{i}, b^{2}=a^{2} x^{j}, c^{4}=$ $\left.x^{k},[a, b]=a^{2} x^{l},[a, c]=x^{m},[b, c]=x^{n}, x^{2}=1,[x, a]=[x, b]=[x, c]=1\right\rangle$.

It is easy to prove the following facts:

1. $o(a)=4$;

2. $G^{\prime} \cong C_{2} \times C_{2}$ and $m, n$ are not 0 in the same time.

If $k=0$, then $G=\langle a, b, c| a^{4}=1, b^{2}=a^{2} x^{j}, c^{4}=1,[a, b]=a^{2} x^{l},[a, c]=$ $\left.x^{m},[b, c]=x^{n}, x^{2}=1,[x, a]=[x, b]=[x, c]=1\right\rangle$. Let $H=\left\langle a^{n} b^{m}, c\right\rangle \cong C_{4} \times C_{4}$. Then $|H|=2^{4}$. Obviously, $H \nsubseteq G$. But $|G: H|=p^{2}$. This contradicts Lemma 4.1 .

If $k=1$, then $G=\langle a, b, c| a^{4}=1, b^{2}=a^{2} x^{j}, c^{4}=x,[a, b]=a^{2} x^{l},[a, c]=$ $\left.x^{m},[b, c]=x^{n}, x^{2}=1,[x, a]=[x, b]=[x, c]=1\right\rangle$. Let $H=\left\langle a^{n} b^{m}\right\rangle \cong C_{4}$. Then 
$|H|=2^{2}$. Obviously, $H \nsubseteq G$. But $N_{G}(H) \geq\left\langle a^{n} b^{m}, c\right\rangle \cong C_{4} \times C_{8}$. It follows that $N_{G}(H) \cong C_{4} \times C_{8}$. Thus $\left|N_{G}(H): H\right|=2^{3}$, a contradiction.

Case 5: If $G / N=\langle\bar{a}, \bar{b}, \bar{c}| \bar{a}^{4}=\bar{b}^{4}=1, \bar{c}^{2}=\bar{b}^{2},[\bar{a}, \bar{b}]=\overline{1},[\bar{a}, \bar{c}]=\bar{b}^{2},[\bar{b}, \bar{c}]=$ $\left.\bar{a}^{2}\right\rangle$. Assume $N=\langle x\rangle$. Then $G=\langle a, b, c| a^{4}=x^{i}, b^{4}=x^{j}, c^{2}=b^{2} x^{k},[a, b]=$ $\left.x^{m},[a, c]=b^{2} x^{n},[b, c]=a^{2} x^{l}, x^{2}=1,[x, a]=[x, b]=[x, c]=1\right\rangle$.

It is easy to prove the following facts:

1. $o(a)=4$ and $o(b)=4$;

2. $G^{\prime} \cong C_{2}^{3}$ and $m=1$;

3. $c(G)=2$;

4. $\Omega_{1}(G)=\left\langle a^{2}, b^{2}, c^{2}\right\rangle \leq Z(G)$.

By the above facts we can assume $G=\langle a, b, c| a^{4}=1, b^{4}=1, c^{2}=b^{2} x^{k}$, $\left.[a, b]=x,[a, c]=b^{2} x^{n},[b, c]=a^{2} x^{l}, x^{2}=1,[x, a]=[x, b]=[x, c]=1\right\rangle$. By discussing the possible values for $k, n, l$, we know there exists $H \cong M_{2}(2,2)$, and $H \unlhd G$. But $|G: H|=2^{2}$. This contradicts Lemma 4.1.

Case 6: If $G / N \cong\langle\bar{a}, \bar{b}, \bar{c}| \bar{a}^{4}=\bar{b}^{4}=\bar{c}^{4}=1,[\bar{b}, \bar{c}]=\bar{a}^{2} \bar{b}^{2},[\bar{a}, \bar{b}]=\bar{c}^{2},[\bar{a}, \bar{c}]=$ $\left.\bar{b}^{2} \bar{c}^{2},\left[\bar{c}^{2}, \bar{a}\right]=1,\left[\bar{c}^{2}, \bar{b}\right]=1\right\rangle$. Assume $N=\langle x\rangle$. Then $G=\langle a, b, c| a^{4}=x^{i}, b^{4}=$ $x^{j}, c^{4}=x^{k},[b, c]=a^{2} b^{2} x^{l},[a, b]=c^{2} x^{m},[a, c]=b^{2} c^{2} x^{n},\left[c^{2}, a\right]=x^{s},\left[c^{2}, b\right]=$ $\left.x^{t}, x^{2}=1,[x, a]=[x, b]=[x, c]=1\right\rangle$.

It is easy to prove the following facts:

1. $o(a)=4$

2. $o(b)=o(c)=4$;

3. $G^{\prime}=\left\langle a^{2}\right\rangle \times\left\langle b^{2}\right\rangle \times\left\langle c^{2}\right\rangle \times\langle x\rangle \cong C_{2}^{4}$.

By above facts we can assume $G=\langle a, b, c| a^{4}=1, b^{4}=1, c^{4}=1,[b, c]=$ $a^{2} b^{2} x^{l},[a, b]=c^{2} x^{m},[a, c]=b^{2} c^{2} x^{n},\left[c^{2}, a\right]=x^{s},\left[c^{2}, b\right]=x^{t}, x^{2}=1,[x, a]=$ $[x, b]=[x, c]=1\rangle$. If $s=t=0$, then, letting $H=\langle a\rangle$, we have $H \nsubseteq G$. But $N_{G}(H) \geq\left\langle a, b^{2}, c^{2}, x\right\rangle$. It follows that $\left|N_{G}(H): H\right| \geq 2^{3}$, a contradiction. If $s$ and $t$ are not zero in the same time, then, by letting $H=\left\langle c^{2}\right\rangle$, we have $H \unlhd G$. But $N_{G}(H) \geq\left\langle a^{2}, b^{2}, c, x\right\rangle$. It follows that $\left|N_{G}(H): H\right| \geq 2^{3}$, a contradiction.

Case 7: If $G / N$ is abelian and $G$ is not abelian, then $\left|G^{\prime}\right|=p$. By Lemma $2.7, G \cong A_{1} * A_{2} * \cdots * A_{s} Z(G)$. Moreover, assume $G=A_{1} * K Z(G)$. If $K \neq 1$, assume $H \not A_{1}$, then $H \not G$. $\left|N_{A_{1}}(H): H\right| \geq p$. We observed $K \leq N_{G}(H), K \cap A_{1} \leq Z(K)$ and $N_{G}(H) \geq N_{A_{1}}(H) * K$. Thus $\mid N_{G}(H)$ : $H|\geq| N_{A_{1}}(H) K / H \mid=\frac{\left|N_{A_{1}}(H)\right||K|}{\left|N_{A_{1}}(H) \cap K\right||H|} \geq \frac{\left|N_{A_{1}}(H)\right||K|}{\left|A_{1} \cap K\right||H|} \geq \frac{\left|N_{A_{1}}(H)\right||K|}{|Z(K)||H|} \geq p^{3}$, a contradiction. Thus $K=1$. It follows that $G=A_{1} Z(G)$.

If $Z(G) \leq A_{1}$, then $G=A_{1}$. Thus $G \cong$ the group of type (1) by Lemma 4.2 .

If $Z(G) \not A_{1}$, then there exists $g \in Z(G) \backslash A_{1}$ and $g \in N_{G}(H)$. If $H \unlhd A_{1}$ and $\left|N_{A_{1}}(H): H\right| \geq p^{2}$, then $\left|N_{G}(H): H\right| \geq p^{3}$. Thus $G \notin S_{2}$. It follows that $\left|N_{A_{1}}(H): H\right|=p$. By Lemma 3.4, $A_{1} \cong M_{p}(2,1), M_{p}(1,1,1)$ or $M_{p}(2,2)$.

If $A_{1} \cong M_{p}(2,1)$ or $M_{p}(1,1,1)$, then $|Z(G)| \geq p^{2}$ since $\left|Z\left(A_{1}\right)\right|=p$. On the other hand, there exists $H \nsupseteq A_{1}$ and $H \leq A_{1}$. Thus $p^{2}=\left|N_{G}(H): H\right| \geq$ $\frac{\left|N_{A_{1}}(H)\right||Z(G)|}{\left|Z\left(A_{1}\right)\right||H|} \geq|Z(G)|$. It follows that $|Z(G)|=p^{2}$. If $Z(G) \cong C_{p} \times C_{p}$, 
then $G \cong M_{p}(2,1) \times C_{p}$ or $G \cong M_{p}(1,1,1) \times C_{p}$. By Lemma $4.3, G \notin S_{2}$. If $Z(G) \cong C_{p^{2}}$, then $G \cong$ the group of type (2).

If $A_{1} \cong M_{p}(2,2)$, then $|Z(G)|=p^{3}$ by a similar argument as above paragraph. Since $Z\left(A_{1}\right) \cong C_{p} \times C_{p} \leq Z(G), Z(G) \cong C_{p} \times C_{p} \times C_{p}$ or $Z(G) \cong$ $C_{p^{2}} \times C_{p}$.

If $Z(G) \cong C_{p} \times C_{p} \times C_{p}$, then $G \cong M_{p}(2,2) \times C_{p}$. By Lemma $4.2, G \notin S_{2}$.

If $Z(G) \cong C_{p^{2}} \times C_{p}$, then $G \cong M_{p}(2,2) * C_{p^{2}}=\langle a, b, c\rangle=\left\langle a^{p^{2}}=1, b^{p^{2}}=\right.$ $\left.1, c^{p^{2}}=1,[a, b]=a^{p},[a, c]=[b, c]=1, c^{p}=a^{i p b^{j p}}\right\rangle$. If $j \not \equiv 0(\bmod p)$, then, by letting $b_{1}=a^{i} b^{j}, a_{1}=a^{j}$, we have $G=\left\langle a_{1}, b_{1}, c\right\rangle=\left\langle a_{1}{ }^{p^{2}}=1, b_{1}{ }^{2}=\right.$ $\left.1, c^{p^{2}}=1,\left[a_{1}, b_{1}\right]=a_{1}^{p},\left[a_{1}, c\right]=\left[b_{1}, c\right]=1, c^{p}=b_{1}{ }^{p}\right\rangle$. Let $H=\left\langle b_{1}, c\right\rangle$. Then $H \not G$. But $|G: H|=p^{2}$. This contradicts Lemma 4.1. If $j \equiv 0(\bmod p)$, then, letting $H=\left\langle c a^{-i}\right\rangle$. Obviously, $H \nsubseteq G$. But $N_{G}(H)=\left\langle a, c, b^{p}\right\rangle$. Thus $\left|N_{G}(H): H\right|=p^{3}$, a contradiction. That means $G \notin S_{2}$.

If $G / N \cong Q_{8} \times C_{2}$, then $\left|G^{\prime}\right|=2^{2}$. Assume $N=\langle x\rangle$. Then $G=$ $\langle a, b, c, x| a^{4}=x^{i}, b^{2}=a^{2} x^{j}, c^{2}=x^{k},[a, b]=a^{2} x^{l},[a, c]=x^{m},[b, c]=x^{n}, x^{2}=$ $1,[x, a]=[x, b]=[x, c]=1\rangle$.

Since $a^{-2} b^{2} \in Z(G),\left[a^{-2} b^{2}, b\right]=1$. we get by calculation $\left[a^{-2} b^{2}, b\right]=$ $\left[a^{-2}, b\right]^{b^{2}}=\left([a, b]^{-2}\right)^{b^{2}}=a^{-4}=1$. Thus $o(a)=4$. Since $\bar{G}^{\prime}=\left\langle\overline{a^{2}}\right\rangle, G^{\prime}=$ $\left\langle a^{2}, x\right\rangle=\left\langle a^{2}\right\rangle \times\langle x\rangle$. Moreover, $m, n$ are not zero in the same time. Since $G^{\prime} \leq Z(G), c(G)=2$. So we can assume $G=\langle a, b, c, x| a^{4}=1, b^{2}=a^{2} x^{j}, c^{2}=$ $\left.x^{k},[a, b]=a^{2} x^{l},[a, c]=x^{m},[b, c]=x^{n}, x^{2}=1,[x, a]=[x, b]=[x, c]=1\right\rangle$.

If $k=0$, then there exists $H \cong C_{4} \times C_{2}$. It is easy to see that $H \nsubseteq G$. But $|G: H|=p^{2}$. This contradicts Lemma 4.1. If $k=1$, then $G \cong$ the group of type (5) by discussing the possible values for $j, l, m, n$.

$\Longleftarrow$ : Case 1 : If $G \cong$ the group of type (1), then the conclusion is true by Lemma 4.2.

Case 2: If $G \cong$ the group of type (2), that is, $G=\langle a, b, c| a^{p^{2}}=b^{p}=c^{p}=$ $\left.1,[b, c]=a^{p},[a, b]=[a, c]=1\right\rangle \cong M_{p}(1,1,1) * C_{p^{2}}$, then $Z(G)=\langle a\rangle, G^{\prime}=\left\langle a^{p}\right\rangle$. If $|H|=p^{3}$, then $H \lessdot G$. Thus $H \unlhd G$. If $|H|=p^{2}$, then $|H \cap\langle a\rangle| \neq 1$. It follows that $G^{\prime} \leq H$, so $H \unlhd G$. If $|H|=p$ and $H \unlhd G$, then $N_{G}(H) \geq\langle H, Z(G)\rangle$. Thus $\left|N_{G}(H)\right| \geq p^{3}$. It follows that $\left|N_{G}(H)\right|=p^{3}$. So $\left|N_{G}(H): H\right|=p^{2}$. That means $G \in \mathcal{S}_{2}$.

Case 3: If $G \cong$ the group of type (3), where $p>2$, then $G=\langle a, b, c| a^{p^{2}}=$ $\left.b^{p^{2}}=c^{p}=1,[a, b]=1,[a, c]=b^{k p},[b, c]=a^{p} b^{h p}\right\rangle, k+4^{-1} h^{2}$ is a fixed quadratic non-residue $(\bmod p)$, where $k=1$ or $\nu, \nu$ is a fixed quadratic non-residue (mod $p), h=0,1, \ldots, \frac{p-1}{2}$, then $G^{\prime}=\left\langle a^{p} b^{h p}, b^{k p}\right\rangle, Z(G)=\Phi(G)=\left\langle a^{p}\right\rangle \times\left\langle b^{p}\right\rangle, G_{3}=$ $1, c(G)=2$, and $G$ is $p$-abelian.

It is easy to prove that all quotient groups of order $p^{4}$ of $G$ are isomorphic to $M_{p}(1,1,1) * C_{p^{2}}$.

For any $H \not g G$, we prove $\left|N_{G}(H): H\right|=p^{2}$ as follows. So $G \in \mathcal{S}_{2}$.

If $|H|=p^{3}$, then $\left|H \cap G^{\prime}\right| \leq p$. If $H \cap G^{\prime}=1$, since $N_{G}(H) \geq\left\langle H, G^{\prime}\right\rangle$ and $|G|=\left|\left\langle H, G^{\prime}\right\rangle\right|, H \unlhd G$. This is a contradiction. If $\left|H \cap G^{\prime}\right|=p$, let 
$\bar{G}=G / H \cap G^{\prime}$. Since $|\bar{G}|=p^{4}, \bar{G} \cong M_{p}(1,1,1) * C_{p^{2}}$. Since $|\bar{H}|=p^{2}$, by using the result of Case 2 , we have $\mid \bar{H} \unlhd \bar{G}$. Thus $H \unlhd G$, a contradiction.

If $|H|=p^{2}$, then $\left|H \cap G^{\prime}\right| \leq p$. If $\left|H \cap G^{\prime}\right|=1$, then $N_{G}(H) \geq\left\langle H, G^{\prime}\right\rangle$. Since $H \notin G,\left|N_{G}(H)\right|=p^{4}$. Thus $\left|N_{G}(H): H\right|=p^{2}$. If $\left|H \cap G^{\prime}\right|=p$, let $\bar{G}=G / H \cap G^{\prime}$. Since $|\bar{G}|=p^{4}, \bar{G} \cong M_{p}(1,1,1) * C_{p^{2}}$. If $H \not G$, then $\bar{H} \notin \bar{G}$. By using the result of Case 2, we have $\left|N_{\bar{G}}(\bar{H}): \bar{H}\right|=p^{2}$. Thus $\left|N_{G}(H): H\right|=\left|N_{\bar{G}}(\bar{H}): \bar{H}\right|=p^{2}$.

If $|H|=p$, since $\Omega_{1}(G)=\left\langle a^{p}\right\rangle \times\left\langle b^{p}\right\rangle \times\langle c\rangle$, assume $H=\left\langle a^{i p} b^{j p} c^{k^{\prime}}\right\rangle$. If $k^{\prime} \equiv$ $0(\bmod p)$, then $H \unlhd G$, a contradiction. Thus $k^{\prime} \neq \equiv 0(\bmod p), 0 \leq i, j \leq p-1$. $\left(a^{i p} b^{j p} c^{k^{\prime}}\right)^{a^{s} b^{t} c^{u}}=a^{i p} b^{j p} c^{k^{\prime}} a^{-k^{\prime} t p} b^{-h k^{\prime} t p-k k^{\prime} s p} \in\left\langle a^{i p} b^{j p} c^{k^{\prime}}\right\rangle$. We get

$$
\begin{cases}-k^{\prime} t \equiv 0 & (\bmod p) \\ -h k^{\prime} t-k k^{\prime} s \equiv 0 & (\bmod p)\end{cases}
$$

It follows by $k \not \equiv 0(\bmod p)$ that

$$
\left\{\begin{aligned}
t \equiv 0 & (\bmod p) \\
s \equiv 0 & (\bmod p)
\end{aligned}\right.
$$

Thus $N_{G}(H)=\left\{a^{s} b^{t} c^{u} \mid t \equiv 0(\bmod p), s \equiv 0(\bmod p)\right\}$. So $\left|N_{G}(H)\right|=p^{3}$, and $\left|N_{G}(H): H\right|=p^{2}$.

If $p=2$, then $G \cong\langle a, b, c| a^{4}=1, b^{4}=1, c^{2}=1,[a, b]=1,[a, c]=b^{2},[b, c]=$ $\left.a^{2} b^{2}\right\rangle . G^{\prime}=\left\langle a^{2}\right\rangle \times\left\langle b^{2}\right\rangle=Z(G)$.

It is easy to prove that all quotient groups of order $2^{4}$ of $G$ are isomorphic to $Q_{8} * C_{4}$.

For any $H \notin G$, we prove $\left|N_{G}(H): H\right|=2^{2}$ as follows. So $G \in \mathcal{S}_{2}$.

If $|H|=2^{3}$, then $\left|H \cap G^{\prime}\right| \leq 2$. If $\left|H \cap G^{\prime}\right|=1$, then $H \unlhd G$ by $N_{G}(H) \geq$ $H G^{\prime}=G$, a contradiction. If $\left|H \cap G^{\prime}\right|=2$, let $\bar{G}=G / H \cap G^{\prime}$. Since $|\bar{G}|=2^{4}$, $\bar{G} \cong Q_{8} * C_{4}$. Since $|\bar{H}|=2^{2}$, in the same way as that of Case 2 , we have $\bar{H} \unlhd \bar{G}$. Thus $H \unlhd G$, a contradiction.

If $|H|=2^{2}$, then $\left|H \cap G^{\prime}\right| \leq 2$. If $\left|H \cap G^{\prime}\right|=1$, then $\left|N_{G}(H)\right| \geq\left|H G^{\prime}\right|=2^{4}$. That means $\left|N_{G}(H)\right|=2^{4}$. Thus $\left|N_{G}(H): H\right|=2^{2}$. If $\left|H \cap G^{\prime}\right|=2$, let $\bar{G}=G / H \cap G^{\prime}$. Since $|\bar{G}|=2^{4}, \bar{G} \cong Q_{8} * C_{4}$. But $|\bar{H}|=2$, and $\bar{H} \not \bar{G}$. In the same way as that of Case 2 , we have $\left|N_{\bar{G}}(\bar{H}): \bar{H}\right|=2^{2}$. Thus $\left|N_{G}(H): H\right|=$ $\left|N_{\bar{G}}(\bar{H}): \bar{H}\right|=2^{2}$.

If $|H|=2$, then we determine $H$ and $N_{G}(H)$ as follows.

For any $g \in G$, we have $g=a^{i} b^{j} c^{k}$. If $o(g)=2$, then

$$
\begin{aligned}
\left(a^{i} b^{j} c^{k}\right)^{2} & =\left(a^{i} b^{j}\right)^{2}\left[a^{i} b^{j}, c^{-k}\right] c^{2 k} \\
& =a^{2 i}\left[a^{i}, b^{-j}\right] b^{2 j}\left[a^{i}, c^{-k}\right]\left[b^{j}, c^{-k}\right] c^{2 k} \\
& =a^{2 i} b^{2 j} b^{-2 i k} a^{-2 j k} b^{-2 j k} c^{2 k} \\
& =a^{2(i-j k)} b^{2(j-i k-j k)} c^{2 k}=1 .
\end{aligned}
$$


It follows that

$$
\begin{cases}i-j k \equiv 0 & (\bmod 2) \\ j-i k-j k \equiv 0 & (\bmod 2)\end{cases}
$$

Moreover,

$$
\left\{\begin{array}{cc}
i \equiv 0 & (\bmod 2) \\
j \equiv 0 & (\bmod 2)
\end{array}\right.
$$

So we can assume $H=\left\langle a^{2 i} b^{2 j} c^{k}\right\rangle$. If $k \equiv 0(\bmod 2)$, then $H \unlhd G$, a contradiction. Thus $k \not \equiv 0(\bmod 2), i, j=0,1$.

Assume $a^{s} b^{t} c^{u} \in N_{G}(H)$. Then $\left(a^{2 i} b^{2 j} c^{k}\right)^{a^{s} b^{t} c^{u}}=a^{2 i} b^{2 j}\left(c^{k} b^{-2 k s}\right)^{b^{t} c^{u}}=$ $a^{2 i} b^{2 j} c^{k} a^{-2 t k} b^{-2 t k} b^{-2 k s}=a^{2 i} b^{2 j} c^{k} a^{-2 t k} b^{-2 t k-2 k s} \in\left\langle a^{2 i} b^{2 j} c^{k}\right\rangle$. It follows that

$$
\left\{\begin{array}{cc}
t k \equiv 0 & (\bmod 2) \\
k s \equiv 0 & (\bmod 2)
\end{array}\right.
$$

Moreover,

$$
\begin{cases}t \equiv 0 & (\bmod 2) \\ s \equiv 0 & (\bmod 2) .\end{cases}
$$

It follows that $N_{G}(H)=\left\{a^{s} b^{t} c^{u} \mid t \equiv 0(\bmod 2), s \equiv 0(\bmod 2)\right\}$. Thus $\left|N_{G}(H)\right|=2^{3}$, and $\left|N_{G}(H): H\right|=2^{2}$.

Case 4: If $G \cong$ the group of type (4), that is, $G=\langle a, b, c| a^{4}=1, b^{2}=$ $\left.a^{2}, c^{4}=1,[a, b]=a^{2},[a, c]=[b, c]=1\right\rangle$, then $G^{\prime}=\left\langle a^{2}\right\rangle, Z(G)=\left\langle a^{2}\right\rangle \times\langle c\rangle$.

For any $H \not G$, we prove $\left|N_{G}(H): H\right|=2^{2}$ as follows. That means $G \in \mathcal{S}_{2}$.

If $|H|=2^{3}$, then $|H \cap\langle a\rangle| \neq 1$. If not, since $|H \cap\langle a, b\rangle| \geq 2, H \cap\langle a, b\rangle$ must contain an element of order 2. But $\langle a, b\rangle \cong Q_{8}$ has unique element $a^{2}$ of order 2, so $a^{2} \in H$, a contradiction. Thus $H>G^{\prime}$, that means $H \unlhd G$, a contradiction. If $|H|=2^{2}$, then $|H \cap Z(G)| \leq 2$. But $\left|N_{G}(H)\right| \geq|H Z(G)|=$ $\frac{|H||Z(G)|}{|H \cap Z(G)|} \geq 2^{4}$, so $\left|N_{G}(H)\right|=2^{4}$. Thus $\left|N_{G}(H): H\right|=2^{2}$. If $|H|=2$, since $\Omega_{1}(G)=\left\langle a^{2}\right\rangle \times\left\langle c^{2}\right\rangle \leq Z(G), H \unlhd G$, a contradiction.

Case 5: If $G \cong$ the group of type (5), i.e., $G=\langle a, b, c| a^{4}=1, b^{4}=1, c^{2}=$ $\left.b^{2},[a, b]=1,[a, c]=b^{2},[b, c]=a^{2}\right\rangle$, then $G^{\prime}=\left\langle a^{2}\right\rangle \times\left\langle b^{2}\right\rangle=Z(G)=\Omega_{1}(G)$, and so $H \cap G^{\prime} \neq 1$ for any $H \leq G$.

It is easy to prove that all quotient groups of order $p^{4}$ of $G$ are isomorphic to $Q_{8} * C_{4}$ or $Q_{8} \times C_{2}$.

For any $H \not G$, we prove $\left|N_{G}(H): H\right|=2^{2}$ as follows, That means $G \in \mathcal{S}_{2}$.

If $|H|=2^{3}$, then $\left|H \cap G^{\prime}\right|=2$. Let $\bar{G}=G / H \cap G^{\prime}$. Since $|\bar{G}|=2^{4}$, $\bar{G} \cong Q_{8} * C_{4}$ or $Q_{8} \times C_{2}$. If $\bar{G} \cong Q_{8} * C_{4}$, since $|\bar{H}|=2^{2}, \bar{H} \unlhd \bar{G}$ by the same argument as that of Case 2. So $H \unlhd G$, a contradiction. If $\bar{G} \cong Q_{8} \times C_{2}$, then $\bar{H} \unlhd \bar{G}$. That means $H \unlhd G$, a contradiction.

If $|H|=2^{2}$, then $\left|\bar{H} \cap G^{\prime}\right|=2$. Let $\bar{G}=G / H \cap G^{\prime}$. Since $|\bar{G}|=2^{4}$, $\bar{G} \cong Q_{8} * C_{4}$ or $Q_{8} \times C_{2}$. If $\bar{G} \cong Q_{8} * C_{4}$, since $|\bar{H}|=2,\left|N_{\bar{G}}(\bar{H}): \bar{H}\right|=2^{2}$ by the same argument as that of Case 2. Thus $\left|N_{G}(H): H\right|=\left|N_{\bar{G}}(\bar{H}): \bar{H}\right|=2^{2}$. If $\bar{G} \cong Q_{8} \times C_{2}$, then $\bar{H} \unlhd \bar{G}$. So $H \unlhd G$, a contradiction.

If $|H|=2$, since $\Omega_{1}(G)=\left\langle a^{2}\right\rangle \times\left\langle b^{2}\right\rangle \leq Z(G), H \unlhd G$, a contradiction. 
Case 6: If $G \cong$ the group of (6), i.e., $G=\langle a, b, c| a^{4}=1, b^{4}=1, c^{4}=$ $\left.1,[a, b]=c^{2},[a, c]=b^{2} c^{2},[b, c]=a^{2} b^{2},\left[c^{2}, a\right]=\left[c^{2}, b\right]=1\right\rangle$, then $G^{\prime}=\left\langle a^{2}\right\rangle \times$ $\left\langle b^{2}\right\rangle \times\left\langle c^{2}\right\rangle=Z(G)=\Omega_{1}(G)$, and so $H \cap G^{\prime} \neq 1$ for any $H \leq G$.

It is easy to prove that all quotient groups of order $p^{5}$ of $G$ are isomorphic to the group of type (3).

For any $H \not G$, we prove $\left|N_{G}(H): H\right|=2^{2}$ as follows, That means $G \in \mathcal{S}_{2}$. If $|H|=2^{4}$, then $\left|H \cap G^{\prime}\right| \leq 2^{2}$. If $\left|H \cap G^{\prime}\right|=2$, let $\bar{G}=G / H \cap G^{\prime}$. Since $|\bar{G}|=2^{5}, \bar{G} \cong$ the group of type (3), Since $|\bar{H}|=2^{3}, \bar{H} \unlhd \bar{G}$ by the same argument as that of Case 3, a contradiction. If $\left|H \cap G^{\prime}\right|=2^{2}$, then there exists $N \leq H \cap G^{\prime}$ and $|N|=2$ such that $G / H \cap G^{\prime} \cong G / N / H \cap G^{\prime} / N$. Since $G / N \cong$ the group of type (3), $G / H \cap G^{\prime} \cong$ the group of type (2) by the same argument as that of Case 3 , Since $|\bar{H}|=2^{2}, \bar{H} \unlhd \bar{G}$ by the same argument as that of Case 2. So $H \unlhd G$, a contradiction.

If $|H|=2^{3}$, then $\left|H \cap G^{\prime}\right| \leq 2^{2}$. If $\left|H \cap G^{\prime}\right|=2$, let $\bar{G}=G / H \cap G^{\prime}$. Since $|\bar{G}|=2^{5}, \bar{G} \cong$ the group of type (3). Since $|\bar{H}|=2^{2}$ and $\bar{H} \sharp \bar{G}$, $\left|N_{\bar{G}}(\bar{H}): \bar{H}\right|=2^{2}$ by the same argument as that of Case 3. Thus $\mid N_{G}(H)$ : $H|=| N_{\bar{G}}(\bar{H}): \bar{H} \mid=2^{2}$. If $\left|H \cap G^{\prime}\right|=2^{2}$, then there exists $N \leq H \cap G^{\prime}$ and $|N|=2$, such that $G / H \cap G^{\prime} \cong G / N / H \cap G^{\prime} / N$. Since $G / N \cong$ the group of type (3), $G / H \cap G^{\prime} \cong$ the group of type (2) by the same argument as that of Case 3. Let $\bar{G}=G / H \cap G^{\prime}$ and $|\bar{H}|=2$. Since $\bar{H} \notin \bar{G},\left|N_{\bar{G}}(\bar{H}): \bar{H}\right|=2^{2}$ by the result of Case 2. Thus $\left|N_{G}(H): H\right|=\left|N_{\bar{G}}(\bar{H}): \bar{H}\right|=2^{2}$.

If $|H|=2^{2}$, then $\left|H \cap G^{\prime}\right|=2$. Let $\bar{G}=G / H \cap G^{\prime}$. Since $|\bar{G}|=2^{5}, \bar{G} \cong$ the group of type (3). Since $|\bar{H}|=2$ and $\bar{H} \not \bar{G},\left|N_{\bar{G}}(\bar{H}): \bar{H}\right|=2^{2}$ by the result of Case 3. Thus $\left|N_{G}(H): H\right|=\left|N_{\bar{G}}(\bar{H}): \bar{H}\right|=2^{2}$.

If $|H|=2$, since $\Omega_{1}(G)=\left\langle a^{2}\right\rangle \times\left\langle b^{2}\right\rangle \times\left\langle c^{2}\right\rangle \leq Z(G), H \unlhd G$, a contradiction.

The groups listed in theorem are mutually non-isomorphic, the details are omitted.

\section{Classifying $\mathcal{S}_{3}$}

Theorem 5.1. If $G$ is a non-Dedekind p-group, then $G \in \mathcal{S}_{3}$ if and only if $G$ is one of the following mutually non-isomorphic groups

(1) $M_{p}(i+1, m)$, where $m \leq i+1$;

(2) $M_{p}(1,1,1) * C_{p^{i}}$

(3) $D_{8} * Q_{8},(i=3)$;

(4) $\langle a, b, c, d| a^{4}=b^{4}=c^{4}=d^{4}=1, a^{2}=d^{2}, b^{2}=c^{2},[d, b]=a^{2},[b, a]=$ $\left.a^{2},[c, a]=b^{2},[d, a]=[c, b]=a^{2} b^{2},[c, d]=1\right\rangle,(i=3)$.

Proof. $\Longrightarrow$ : Case 1. $\left|G^{\prime}\right|=p$.

Let $N_{1}$ be a non-normal subgroup of $G$ with minimal order, then all of maximal subgroups of $N_{1}$ are normal in $G$, and $N_{1}$ is nonnormal in $G$. Then $N_{1}$ cannot be generated by its maximal subgroups, the maximal subgroup of $N_{1}$ is unique, thus $N_{1}$ is cyclic. Let $N_{1}=\langle b\rangle$. Since $N_{1}$ is non-normal in $G$, there exists $a \in G$ such that $[a, b] \neq 1$. Because $\left|G^{\prime}\right|=p$, we have $\langle a, b\rangle$ is 
a minimal nonabelian subgroup of $G$. Let $H=\langle a, b\rangle$. By Lemma 2.10, we obtain $G=H * C_{G}(H)$. Since $H$ is minimal nonabelian, we have $C_{H}\left(N_{1}\right) \lessdot H$. And $C_{G}\left(N_{1}\right) \geq C_{G}(H), C_{G}\left(N_{1}\right) \geq C_{H}\left(N_{1}\right)$, thus $C_{G}\left(N_{1}\right)=N_{G}\left(N_{1}\right) \lessdot G$. By $G \in \mathcal{S}_{3}$, we can get $N_{1}$ is a nonnormal subgroup of $G$ with maximal order, it follows that all nonnormal subgroups of $G$ are of same order.

If all nonnormal subgroups of $G$ are of order $p$, by Lemma 2.9, $G$ is one of following groups:

(1) $M_{p}(i+1,1)$;

(2) $M_{p}(1,1,1) * C_{p^{i}}$

(3) $D_{8} * Q_{8}(\mathrm{i}=3)$.

If all of nonnormal subgroups of $G$ are of order $p^{m}$, where $m \geq 2$, then $\Omega_{1}(G) \leq Z(G)$. When $p>2$, by Lemma 2.8 , we get $G \cong M_{p}(i+1, m)$, where $m \leq i+1$. When $p=2$, since $G=H * C_{G}(H), G \in \mathcal{S}_{3}$, so is $H$, we can get $H \cong M_{2}(i+1, m)$, where $m \leq i+1$. Assume that $C_{G}(H) \nless H$, then there exists $c \in C_{G}(H) \backslash H$. Let $H=\langle a\rangle \rtimes\langle b\rangle$ and $c^{2^{n}}=a^{2^{s}} b^{2^{t}}, n, s, t \geq 1$. We get a contradiction, thus $C_{G}(H) \leq H, G \cong M_{2}(i+1, m)$, where $m \leq i+1$.

If $s \geq 2$, we have $c_{1}=c^{-2^{n-1}} a^{2^{s-1}} b^{2^{t-1}} \notin H$ with order $p$, then $\left\langle b, c_{1}\right\rangle \nsupseteq G$ and $\left|\left\langle b, c_{1}\right\rangle\right| \neq|\langle b\rangle|$, which is contrary to that all of nonnormal subgroups of $G$ are of same order.

If $s=1$ and $t \geq 2$, we have $c_{1}=c^{-2^{n-1}} a b^{2^{t-1}} \notin H$ with order $p$, then $\left\langle c_{1}\right\rangle \not \nexists G$ and $\left|\left\langle c_{1}\right\rangle\right| \neq|\langle b\rangle|$, which is contrary to that all nonnormal subgroups of $G$ are of same order.

If $s=1$ and $t=1$, let $K=\langle H, c\rangle=\left\langle a^{2^{i+1}}=b^{2^{m}}=1,[a, b]=a^{2^{i}}, c^{2^{n}}=\right.$ $\left.a^{2} b^{2},[c, a]=[c, b]=1\right\rangle$, where $m \leq i+1$. If $i+1 \geq 3$, we have $c_{1}=$ $c^{-2^{n-1}} a b a^{2^{i}} \notin H$ with $c_{1}=c^{-2^{n-1}} a b a^{2^{i}} \notin H$ with order $p$, then $\left\langle c_{1}\right\rangle \nsupseteq G$ and $\left|\left\langle c_{1}\right\rangle\right| \neq|\langle b\rangle|$, which is contrary to that all nonnormal subgroups of $G$ are of order $\left|N_{1}\right|$. If $i+1=m=2$, since $G \in \mathcal{S}_{3}$ and $\langle b\rangle \nsupseteq G$, we have $n \geq 2$ thus $\langle c a\rangle \not G$, and $|\langle c a\rangle| \neq|\langle b\rangle|$, which is contrary to that all nonnormal subgroups of $G$ are of same order.

Case 2. $\left|G^{\prime}\right| \geq p^{2}$.

We use induction on $|G|$. If $|G|=p^{5}$ and $G \in \mathcal{S}_{3}$, then all nonnormal subgroups of $G$ are of order $p$, by Lemma 2.9 , we can get $G \cong M_{p}(4,1)$, $M_{p}(1,1,1) * C_{p^{3}}$ or $D_{8} * Q_{8}$. The conclusion is true. Assume the conclusion is true for groups of order $<|G|$. Since $G$ is a $p$-group, there exists $N \leq G^{\prime} \cap Z(G)$ and $|N|=p$. By Lemma 2.13 and $|G / N|<|G|, G / N$ is the group of listed in Theorem by induction hypothesis.

If $G / N \cong M_{p}(i+1, m)=\left\langle\bar{a}, \bar{b} \mid \bar{a}^{p^{i+1}}=1, \bar{b}^{p^{m}}=1,[\bar{a}, \bar{b}]=\bar{a}^{p^{i}}\right\rangle$, then it follows by $\left|(G / N)^{\prime}\right|=\left|G^{\prime} N / N\right|=\left|G^{\prime} / G^{\prime} \cap N\right|=\left|G^{\prime} / N\right|=p$ that $\left|G^{\prime}\right|=p^{2}$. By Lemma $2.3, G \cong\left\langle a, b \mid a^{p^{i+2}}=1, b^{p^{m}}=1,[a, b]=a^{p^{i}}\right\rangle$, where $m \geq 2$. Let $H=\left\langle b^{p}\right\rangle$. Obviously, $H \not G$. But $N_{G}(H) \geq\left\langle a^{p}, b\right\rangle$. Thus $\left|N_{G}(H): H\right| \geq$ $p^{i+2}$, a contradiction. 
If $G / N \cong M_{p}(1,1,1) * C_{p^{i}}=\langle\bar{a}, \bar{b}, \bar{c}\rangle=\left\langle\bar{a}^{p^{i}}=1, \bar{b}^{p}=1, \bar{c}^{p}=1,[\bar{b}, \bar{c}]=\right.$ $\left.\bar{a}^{p^{i-1}},[\bar{a}, \bar{b}]=1,[\bar{a}, \bar{c}]=1\right\rangle$, then, letting $N=\langle x\rangle, G=\langle a, b, c| a^{p^{i}}=x^{s}, b^{p}=$ $x^{j}, c^{p}=x^{k},[a, b]=x^{l},[a, c]=x^{m},[b, c]=a^{p^{i-1}} x^{n}, x^{p}=1,[x, a]=[x, b]=$ $[x, c]=1\rangle$. Since $\left|(G / N)^{\prime}\right|=\left|G^{\prime} N / N\right|=\left|G^{\prime} / G^{\prime} \cap N\right|=\left|G^{\prime} / N\right|=p,\left|G^{\prime}\right|=p^{2}$. Thus $G$ is metaabelian. Since $b^{p} \in Z(G), 1=\left[b^{p}, c\right]=[b, c]^{p}[b, c, b]^{\left(\begin{array}{c}p \\ 2\end{array}\right)}=$

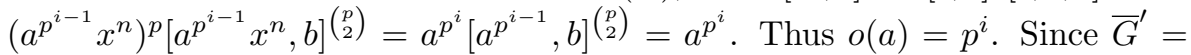
$\overline{G^{\prime}}=\left\langle\overline{a^{p^{i-1}}}\right\rangle, G^{\prime}=\left\langle a^{p^{i-1}}, x\right\rangle \cong C_{p} \times C_{p}$. Moreover, $l, m$ are not zero in the same time. Assume $G=\langle a, b, c| a^{p^{i}}=1, b^{p}=x^{j}, c^{p}=x^{k},[a, b]=x^{l},[a, c]=$ $\left.x^{m},[b, c]=a^{p^{i-1}} x^{n}, x^{p}=1,[x, a]=[x, b]=[x, c]=1\right\rangle$. Let $H=\langle a\rangle$. Since $l, m$ are not zero in the same time, $H \notin G$. But $\left|N_{G}(H)\right| \leq p^{i+2}$. Thus $\left|N_{G}(H): H\right| \leq p^{2} \neq p^{i}$, a contradiction.

If $G / N \cong D_{8} * Q_{8}=\langle a, b, c, d| \bar{a}^{4}=1, \bar{b}^{2}=1, \bar{c}^{4}=1, \bar{c}^{2}=\bar{d}^{2}, \bar{a}^{2}=$ $\left.\bar{c}^{2},[\bar{a}, \bar{b}]=\bar{a}^{2},[\bar{c}, \bar{d}]=\bar{c}^{2},[\bar{a}, \bar{c}]=[\bar{a}, \bar{d}]=[\bar{b}, \bar{c}]=[\bar{b}, \bar{d}]=1\right\rangle$, then, letting $N=\langle x\rangle, G=\langle a, b, c, d| a^{4}=x^{i}, b^{2}=x^{j}, c^{4}=x^{k}, c^{2}=d^{2} x^{l}, a^{2}=c^{2} x^{m},[a, b]=$ $a^{2} x^{n},[c, d]=c^{2} x^{s},[a, c]=x^{t},[a, d]=x^{u},[b, c]=x^{v},[b, d]=x^{w}, x^{2}=1,[x, a]=$ $[x, b]=[x, c]=[x, d]=1\rangle$. Since $d^{-2} c^{2} \in Z(G),\left[d^{-2} c^{2}, d\right]=1$. On the other hand, $\left[d^{-2} c^{2}, d\right]=\left[c^{2}, d\right]=[c, d]^{2}[c, d, c]=c^{4}$. So $c^{4}=1$. Since $a^{2}=c^{2} x^{m}$, $a^{4}=c^{4}$. Assume $G=\langle a, b, c, d| a^{4}=1, b^{2}=x^{j}, c^{4}=1, c^{2}=d^{2} x^{l}, a^{2}=$ $c^{2} x^{m},[a, b]=a^{2} x^{n},[c, d]=c^{2} x^{s},[a, c]=x^{t},[a, d]=x^{u},[b, c]=x^{v},[b, d]=$ $\left.x^{w}, x^{2}=1,[x, a]=[x, b]=[x, c]=[x, d]=1\right\rangle$. By $\bar{G}^{\prime}=\left\langle\overline{a^{2}}\right\rangle, G^{\prime}=\left\langle a^{2}, x\right\rangle \cong$ $C_{2} \times C_{2}$. Moreover, $G^{\prime} \leq Z(G)$ and $\exp (G)=4$. By the argument of [15, Lemma 4.5], $G=\langle a, b, c, d| a^{4}=b^{4}=1, c^{2}=a^{2} b^{2}, d^{2}=a^{2},[a, b]=a^{2},[c, d]=$ $\left.a^{2} b^{2},[a, c]=[b, d]=1,[b, c]=[a, d]=b^{2}\right\rangle$. It is easy to prove that $G \cong$ the group of type (4).

If $G / N \cong$ the group of type (4), then, letting $N=\langle x\rangle, G=\langle a, b, c, d| a^{4}=$ $x^{i}, b^{4}=x^{j}, c^{4}=x^{k}, d^{4}=x^{l}, a^{2}=d^{2} x^{m}, b^{2}=c^{2} x^{n},[d, b]=a^{2} x^{s},[b, a]=$ $a^{2} x^{t},[c, a]=b^{2} x^{r},[d, a]=a^{2} b^{2} x^{u},[c, b]=a^{2} b^{2} x^{v},[c, d]=x^{w}, x^{2}=1,[x, a]=$ $[x, b]=[x, c]=[x, d]=1\rangle$. Since $d^{-2} a^{2} \in Z(G),\left[d, d^{-2} a^{2}\right]=1$. On the other hand, $\left[d, d^{-2} a^{2}\right]=\left[d, a^{2}\right]=[d, a]^{2}[d, a, a]=\left(a^{2} b^{2} x^{u}\right)^{2}\left[a^{2} b^{2} x^{u}, a\right]=$ $a^{4}\left[a^{2}, b^{-2}\right] b^{4}\left[b^{2}, a\right]=\left[b^{2}, a\right] a^{4} b^{4}=a^{4} b^{4}[b, a]^{2}[b, a, b]=b^{4}\left[a^{2}, b\right]=b^{4}[a, b]^{2}[a, b, a]$ $=b^{4} a^{-4}$. So $a^{4}=b^{4}$. It follows by $a^{2}=d^{2} x^{m}$ that $a^{4}=d^{4}$. By $b^{2}=c^{2} x^{n}$ we have $b^{4}=c^{4}$. Thus $a^{4}=b^{4}=c^{4}=d^{4}$.

Assume $a^{4}=b^{4}=c^{4}=d^{4}=x$. Then it follows by $[d, b]=a^{2} x^{s}$ that $[d, b]^{a}=\left(a^{2} x^{s}\right)^{a}$. On the other hand,

$$
\begin{aligned}
{[d, b]^{a} } & =\left[d^{a}, b^{a}\right]=\left[d a^{2} b^{2}, b a^{2}\right]=\left[d, b a^{2}\right]^{a^{2} b^{2}}\left[a^{2} b^{2}, b a^{2}\right] \\
& =\left[d, a^{2}\right]^{a^{2} b^{2}}[d, b]^{a^{2} \cdot a^{2} b^{2}}\left[a^{2} b^{2}, a^{2}\right]\left[a^{2} b^{2}, b\right]=\left[d, a^{2}\right][d, b]\left[a^{2}, b\right] \\
& =[d, a]^{2}[d, a, a][d, b][a, b]^{2}[a, b, a]=\left(a^{2} b^{2} x^{u}\right)^{2}\left[a^{2} b^{2} x^{u}, a\right] a^{2} x^{s} a^{-4} \\
& =a^{4}\left[a^{2}, b^{-2}\right] b^{4}\left[b^{2}, a\right] a^{2} x^{s} a^{-4}=a^{4} b^{4}[b, a]^{2}[b, a, b] a^{2} x^{s} a^{-4} \\
& =a^{4} b^{4} a^{4}\left[a^{2}, b\right] a^{2} x^{s} a^{-4}=[a, b]^{2}[a, b, a] a^{2} x^{s}=a^{2} x^{s} x .
\end{aligned}
$$


But $\left(a^{2} x^{s}\right)^{a}=a^{2} x^{s}$, a contradiction. So $a^{4}=b^{4}=c^{4}=d^{4}=1$. Assume $G=\langle a, b, c, d| a^{4}=b^{4}=c^{4}=d^{4}=1, a^{2}=d^{2} x^{m}, b^{2}=c^{2} x^{n},[d, b]=$ $a^{2} x^{s},[b, a]=a^{2} x^{t},[c, a]=b^{2} x^{r},[d, a]=a^{2} b^{2} x^{u},[c, b]=a^{2} b^{2} x^{v},[c, d]=x^{w}, x^{2}=$ $1,[x, a]=[x, b]=[x, c]=[x, d]=1\rangle$. By calculation we have $\Omega_{1}(G)=$ $\left\langle a^{2}, b^{2}, x\right\rangle \leq Z(G)$. If $G \in \mathcal{S}_{3}$, then by $|G|=2^{7}$ we have $H \unlhd G$ for any $H \leq G$ and $|H| \geq 2^{4}$. If $|H|=2^{3}$, then $H=\Omega_{1}(G)$ if $\exp (H)=2$, and $H$ is abelian if $\exp (H)=2^{2}$ (If not, $H \cong M_{2}(2,1)$, this contradicts $\Omega_{1}(G) \leq Z(G)$ ). It follows that $G$ is a meta-Hamilton $p$-group. But by checking the classification of meta-Hamilton $p$-groups [1] we know there does not exists such a group, a contradiction.

$\Longleftarrow$ : By Lemmas 2.8, 2.9 we have $G \in \mathcal{S}_{3}$ for $G \cong$ one of the groups of type (1), (2), and (3). If $G \cong$ the group of type (4), then $G^{\prime}=\left\langle a^{2}\right\rangle \times\left\langle b^{2}\right\rangle=Z(G)=$ $\Omega_{1}(G)$.

It is easy to prove that all quotient groups of order $2^{5}$ of $G$ are isomorphic to $Q_{8} * D_{8}$.

For any $H \nsubseteq G$, we prove $\left|N_{G}(H): H\right|=2^{3}$ as follows. Thus $G \in \mathcal{S}_{3}$.

If $|H|=2^{4}$, then $\left|H \cap \Omega_{1}(G)\right|=2$. Let $\bar{G}=G / H \cap \Omega_{1}(G)$. Since $|\bar{G}|=2^{5}$, $\bar{G} \cong$ the group of type (3). But $|\bar{H}|=2^{3}$. It follows by Lemma 2.9 that $\bar{H} \unlhd \bar{G}$. So $H \unlhd G$, a contradiction.

If $|\bar{H}|=2^{3}$, then $\left|H \cap \Omega_{1}(G)\right|=2$. Let $\bar{G}=G / H \cap \Omega_{1}(G)$. Since $|\bar{G}|=2^{5}$, $\bar{G} \cong$ the group of type (3). But $|\bar{H}|=2^{2}$. It follows by Lemma 2.9 that $\bar{H} \unlhd \bar{G}$. So $H \unlhd G$, a contradiction.

If $|\bar{H}|=2^{2}$, then $\left|H \cap \Omega_{1}(G)\right|=2$. Let $\bar{G}=G / H \cap \Omega_{1}(G)$. Since $|\bar{G}|=2^{5}$, $\bar{G} \cong$ the group of type (3). But $|\bar{H}|=2$. It follows by $H \nsubseteq G$ that $\bar{H} \nsupseteq \bar{G}$. By Lemma 2.9 we have $\left|N_{\bar{G}}(\bar{H}): \bar{H}\right|=2^{3}$. Thus $\left|N_{G}(H): H\right|=\left|N_{\bar{G}}(\bar{H}): \bar{H}\right|=$ $2^{3}$.

If $|H|=2$, since $\Omega_{1}(G)=\left\langle a^{2}\right\rangle \times\left\langle b^{2}\right\rangle=Z(G), H \unlhd G$, a contradiction.

Acknowledgements. We cordially thank the referee for her(his) comments.

\section{References}

[1] L. J. An, A classification of finite meta-Hamilton p-groups, Ph. D. dissertation, Beijing University, 2009.

[2] Y. Berkovich, Groups of Prime Power Order. Vol. 1, With a foreword by Zvonimir Janko. de Gruyter Expositions in Mathematics, 46. Walter de Gruyter GmbH \& Co. KG, Berlin, 2008.

[3] R. W. Carter, Nilpotent self-normalizing subgroups of soluble groups, Math. Z. 75 (1960/1961), 136-139.

[4] A. Fattahi, Groups with only normal and abnormal subgroups, J. Algebra 28 (1974), $15-19$.

[5] B. Huppert, Endliche Gruppen I, Spriger-Verlag, Berlin, Heidelberg, New York, 1967.

[6] L. L. Li, H. P. Qu, and G. Y. Chen, Central extension of inner abelian pp-groups. I, Acta Math. Sinica (Chin. Ser.) 53 (2010), no. 4, 675-684.

[7] D. S. Passman, Nonnormal subgroups of p-groups, J. Algebra 15 (1970), 352-370.

[8] L. Rédei, Das schiefe Product in der Gruppentheorie, Comment. Math. Helv. 20 (1947), $225-267$. 
[9] M. Y. Xu, A theorem on metabelian p-groups and some consequences, Chin. Ann. Math. Ser. B 5 (1984), no. 1, 1-6.

[10] M. Y. Xu and H. P. Qu, Finite p-Groups, Beijing University Press, Beijing, 2010.

[11] Q. H. Zhang and J. X. Wang, Finite groups with quasi-normal and self-normalizer subgroups, Acta Math. Sinica (Chin. Ser.) 38 (1995), no. 3, 381-385.

[12] Q. H. Zhang, Finite groups with only seminormal and abnormal subgroups, J. Math. Study 29 (1996), no. 4, 10-15.

[13] Finite groups with only ss-quasinormal and abnormal subgroups, Northeast. Math. J. 14 (1998), no. 1, 41-46.

[14] , s-semipermutability and abnormality in finite groups, Comm. Algebra 27 (1999), no. 9, 4515-4524.

[15] Q. H. Zhang, X. Q. Guo, H. P. Qu, and M. Y. Xu, Finite groups which have many normal subgroups, J. Korean Math. Soc. 46 (2009), no. 6, 1165-1178.

QINHAI ZHANG

Department of Mathematics

Shanxi Normal University

Linfen, ShanXi 041004, P. R. China

E-mail address: zhangqh@dns.sxnu.edu.cn

JUAN GAO

Department of Mathematics

Shanxi Normal University

Linfen, Shanxi 041004, P. R. China

E-mail address: zhangqhon@hotmail.com 